\title{
Population Pharmacokinetic Modelling of Roflumilast and Roflumilast $N$-Oxide by Total Phosphodiesterase-4 Inhibitory Activity and Development of a Population Pharmacodynamic-Adverse Event Model
}

\author{
Gezim Lahu, ${ }^{1,2}$ Andreas Hünnemeyer, ${ }^{1}$ Edgar Diletti, ${ }_{1}^{1}$ Martin Elmlinger, ${ }^{1}$ Peter Ruth, ${ }^{2}$ Karl Zech, ${ }^{1}$ Nigel McCracken ${ }^{1}$
} and Axel Facius ${ }^{1}$

1 Department of Pharmacometrics and Pharmacokinetics, Nycomed GmbH, Konstanz, Germany

2 Institute of Pharmacy, University of Tübingen, Tübingen, Germany

\section{Abstract}

Background: Roflumilast is an oral, selective phosphodiesterase (PDE)-4 inhibitor in development for the treatment of chronic obstructive pulmonary disease (COPD). Both roflumilast and its metabolite roflumilast $N$-oxide have anti-inflammatory properties that contribute to overall pharmacological activity. Objectives: To model the pharmacokinetics of roflumilast and roflumilast $N$-oxide, evaluate the influence of potential covariates, use the total PDE4 inhibitory activity (tPDE4i) concept to estimate the combined inhibition of PDE4 by roflumilast and roflumilast $N$-oxide, and use individual estimates of tPDE4i to predict the occurrence of adverse events (AEs) in patients with moderate-to-severe COPD.

Methods: We modelled exposure to roflumilast and roflumilast $N$-oxide ( 21 studies provided the index dataset and five separate studies provided the validation dataset), extended the models to COPD (using data from two studies) and assessed the robustness of the parameter estimates. A parametric bootstrap estimation was used to quantify tPDE4i in subpopulations. We established logistic regression models for each AE occurring in $>2 \%$ of patients in a placebo-controlled trial that achieved a p-value of $<0.2$ in a permutation test. The exposure variables were the area under the plasma concentration-time curve (AUC) of roflumilast, the AUC of roflumilast $N$-oxide and tPDE4i. Individual AUC values were estimated from population models.

Results: Roflumilast pharmacokinetics were modelled with a two-compartment model with first-order absorption including a lag time. A one-compartment model with zero-order absorption was used for roflumilast $N$-oxide. The final models displayed good descriptive and predictive performance with no appreciable systematic trends versus time, dose or study. Posterior predictive checks and robustness analysis showed that the models adequately described the pharmacokinetic parameters and the covariate effects on disposition. For roflumilast, the covariates of sex, smoking and race influenced clearance; and food influenced the absorption rate constant and lag time. For roflumilast $N$-oxide, age, sex and smoking influenced clearance; age, sex and race influenced the fraction metabolized; bodyweight influenced the apparent volume of distribution; and food influenced the apparent duration of formation. The COPD covariate increased the central volume of distribution of roflumilast by $184 \%$ and reduced its clearance by $39 \%$; it also reduced the estimated volume of distribution of roflumilast $N$-oxide by $21 \%$ and reduced its clearance by $7.9 \%$. Compared with the reference population (male, non-smoking, White, healthy, 40-year-old subjects), the relative geometric mean [95\% CI] tPDE4i was higher in patients with COPD $(12.6 \%$ [-6.6, 35.6]), women $(19.3 \%$ [8.2, 31.6]), Black subjects $(42.1 \%$ [16.4, 73.4]), Hispanic subjects $(28.2 \%$ [4.1, 57.9]) and older subjects (e.g. 8.3\% $[-11.2,32.2]$ in 60 -year-olds), and was lower in smokers $(-19.1 \%[-34.0,-0.7])$. Among all possible subgroups in this analysis, the subgroup with maximal tPDE4i comprised elderly, Black, female, non-smoking, COPD patients (tPDE4i 217\% [95\% CI 107, 437] compared with the value in the reference population). The probability of a patient with tPDE4i at the population geometric mean [95\% CI] was $13.0 \%[7.5,18.5]$ for developing diarrhoea, $6.0 \%[2.6,9.4]$ for nausea and $5.1 \%[1.9,8.6]$ for headache. 
Conclusions: Covariate effects have a limited impact on tPDE4i. There was a general association between tPDE4i and the occurrence of common AEs in patients with COPD.

\section{Background}

Roflumilast (3-cyclopropylmethoxy-4-difluoromethoxy- $N$ (3,5-dichloropyrid-4-yl)-benzamide) is a second-generation, selective phosphodiesterase (PDE)-4 inhibitor under development for the treatment of chronic obstructive pulmonary disease (COPD) ${ }^{[1]}$ PDE4 decreases intracellular cyclic adenosine monophosphate levels and, by inhibiting PDE4, roflumilast suppresses inflammatory cell activity. ${ }^{[2-4]}$ Roflumilast improves lung function and reduces exacerbations compared with placebo in patients with moderate-to-severe $\mathrm{COPD},{ }^{[5,6]}$ including those with chronic bronchitis ${ }^{[7]}$ and those who are concomitantly treated with long-acting bronchodilators. ${ }^{[8]}$

Roflumilast is rapidly metabolized to its only active metabolite, roflumilast $N$-oxide, which has specificity and potency similar to those of roflumilast. ${ }^{[1,9,10]}$ The metabolite is estimated to contribute about $90 \%$ of total PDE4 inhibitory activity (tPDE4i), with $10 \%$ attributed to the parent roflumilast. ${ }^{[1,9-11]}$ Roflumilast is converted to roflumilast $N$-oxide by cytochrome P450 (CYP) 3A4 and 1A2 isoenzymes. ${ }^{[12,13]}$ CYP3A4 is primarily responsible for clearance of roflumilast $N$-oxide, with some contribution from CYP2C19 and extrahepatic CYP1A1. The activity of CYP3A4 and CYP1A2 can be affected by covariates such as age and sex ${ }^{[14-16]}$ and whether the individual smokes. ${ }^{[17]}$ These covariates are predicted to affect the pharmacokinetic profile of roflumilast.

The pharmacokinetic profile of roflumilast and its metabolite have been assessed using samples taken across 26 phase I studies, one phase II study and one phase III study. Briefly, roflumilast has linear pharmacokinetics over the dose range of $250-1000 \mu \mathrm{g}^{[9]}$ and is almost completely absorbed after oral administration, with the maximum plasma concentration $\left(\mathrm{C}_{\max }\right)$ being reached within about 1 hour in healthy subjects. ${ }^{[9,18,19]}$ Absolute bioavailability is $79 \%$ when roflumilast is administered as an immediate-release tablet. ${ }^{[20]}$ The apparent effective plasma half-life ranges from 8 to 31 hours (median 17 hours) ${ }^{[9]}$ and steady-state plasma concentrations are achieved after 3-4 days of oral, once-daily dosing. The $\mathrm{C}_{\max }$ of roflumilast $N$-oxide is reached approximately 8 hours after drug intake, ${ }^{[9,13,18,19,21]}$ and the plasma half-life is approximately 30 hours. ${ }^{[9,13,18,21]}$ Steady-state plasma concentrations of roflumilast $\mathrm{N}$-oxide are achieved within 6 days of oral, once-daily dosing. ${ }^{[9]}$ Roflumilast and roflumilast $N$-oxide are excreted as inactive metabolites via the urine (about $70 \%$ of the administered dose).
The activity of both compounds is included in tPDE4i. This composite parameter includes the intrinsic activity, protein binding and exposure of both roflumilast and its primary active metabolite, roflumilast $N$-oxide. ${ }^{[19,22]}$

The therapeutic index of PDE4 inhibitors depends on their relative specificities for PDE4 isoenzymes, of which there are four subtypes in humans: A, B, C and D. The subtypes differ in their distribution and function, with many of the adverse events (AEs) of PDE4 inhibition being associated with inhibition of PDE4D. ${ }^{[23,24]}$ Whereas roflumilast and roflumilast $N$-oxide do not discriminate between the subtypes (with the exception of a slightly lower potency of roflumilast for PDE4C) ${ }^{[1]}$ the firstgeneration PDE4 inhibitor cilomilast showed some subtype selectivity for PDE4D and was associated with clinically significant AEs, which prevented its clinical use. ${ }^{[25-27]}$

The aims of the current analysis were to model the pharmacokinetics of roflumilast and roflumilast $N$-oxide, to evaluate the influence of potential covariates, to use tPDE4i to estimate the combined PDE4 inhibition of roflumilast and roflumilast $N$ oxide, and to use population pharmacokinetic models to evaluate the tPDE4i concept as a predictor of roflumilast tolerability in a population of patients with moderate-to-severe COPD.

\section{Methods}

\section{Study Design}

Data from 21 phase I studies were used as an index dataset for model building, data from five phase I studies were used for model validation, and data from one phase II and one phase III study were used for extension of the model to patients with COPD (see table S-1 in Supplemental Digital Content 1; http://links.adisonline.com/CPZ/A11). The standard dose of roflumilast was given in the majority of studies $(500 \mu \mathrm{g}$ once daily, given orally as a tablet), but single or repeated doses of $250 \mu \mathrm{g}$ were used in some studies in addition to lower and higher doses in a dose-proportionality study and a dose-escalation study (see details in table S-1 in the Supplemental Digital Content). All studies were approved and monitored by institutional review boards and were conducted in accordance with the Declaration of Helsinki (2006). All subjects provided informed consent prior to enrolment. The demographic characteristics of all subjects who contributed observations used for 
modelling are summarized in table S-2 in the Supplemental Digital Content.

\section{Pharmacokinetic Methods}

Across all studies, roflumilast and roflumilast $N$-oxide concentrations in plasma samples were quantified using validated high-performance liquid chromatography systems coupled with tandem mass spectrometry detection (see table S-3 in the Supplemental Digital Content).

\section{Pharmacokinetic Models}

Separate population pharmacokinetic models were developed for roflumilast (7705 observations from 338 subjects) and roflumilast $N$-oxide ( 7112 observations from 298 subjects) using NONMEM $^{\circledR 1}$ nonlinear mixed-effects modelling software. Data from all studies were dose normalized to $500 \mu \mathrm{g}$ for plotting (not modelling). The concentration-time profiles after a single dose and at steady state are shown in figure 1 . The model-building strategy was based on four major steps.

\section{Base Model}

A two-compartment model with first-order absorption (NONMEM $^{\circledR}$ subroutine ADVAN4) was used. Disposition kinetics were modelled for the apparent oral clearance (CL), apparent central volume of distribution $\left(\mathrm{V}_{1}\right)$, apparent intercompartmental clearance $(\mathrm{Q})$ and apparent peripheral volume of distribution $\left(\mathrm{V}_{2}\right)$ [NONMEM ${ }^{\circledR}$ subroutine TRANS4]. An apparent first-order absorption rate constant $\left(\mathrm{k}_{\mathrm{a}}\right)$ and a lagtime $\left(t_{\text {lag }}\right)$ parameter were used to characterize the absorption process. The HYBRID estimation method of NONMEM ${ }^{\circledR}$ was used, where first-order conditional estimation was used to estimate all parameters except for the $t_{\mathrm{lag}}$, for which the first-order estimation method was used. The intra-individual variability was modelled with an additive error on the log-transformed concentration and reported as the approximate coefficient of variation.

To fit roflumilast $N$-oxide concentrations, a one-compartment model with zero-order absorption (NONMEM ${ }^{\circledR}$ subroutine ADVAN1) was used. The disposition kinetics of roflumilast $N$-oxide were modelled, parameterizing for $\mathrm{CL}$ and the apparent volume of distribution $\left(\mathrm{V}_{\mathrm{d}}\right)\left[\mathrm{NONMEM}^{\circledR}\right.$ subroutine TRANS2]. The duration of formation $\left(D_{1}\right)$ and $t_{\text {lag }}$ parameters were used to characterize the zero-order absorption process.
The HYBRID estimation method of NONMEM $^{\circledR}$ was employed where first-order conditional estimation was used to estimate all parameters except for the $\mathrm{D}_{1}$. For the $\mathrm{D}_{1}$, the firstorder estimation method was used.

The apparent fraction absorbed for roflumilast and the fraction metabolized $\left(\mathrm{F}_{\text {rel }}\right)$ for roflumilast $N$-oxide were unidentifiable, as there were no data following intravenous dosing in the datasets. Hence, covariate effects on the $F_{\text {rel }}$ were relative to $F_{\text {rel }}=1$ for the null values of the covariate effects (i.e. fasted, age $=40$ years, female, non-smoker, no alcohol [ethanol] consumption).

For both models, interindividual variability in the pharmacokinetic parameters was modelled using multiplicative exponential random effects of the form (equation 1):

$$
\theta_{\mathrm{i}}=\theta \times \mathrm{e}^{\eta_{\mathrm{i}}}
$$

where $\theta$ is the typical individual (population mean) value of the parameter and $\eta_{i}$ denotes the interindividual random effect accounting for the deviation of the $\mathrm{i}^{\text {th }}$ individual from the typical value, having a mean of zero and variance of $\omega^{2}$. The multivariate vector of interindividual random effects (across parameters within each individual) had the variance-covariance matrix $\Omega$. For both the roflumilast and roflumilast $N$-oxide models, full-block (unstructured) $\Omega$ s were examined. Alternative reduced structures for $\Omega$ s were also evaluated to obtain a stable and parsimonious covariance structure.

\section{Full Models}

For both roflumilast and roflumilast $N$-oxide, covariates were added to the base model simultaneously to form the full models. The dichotomous covariates examined are shown in table S-2 in the Supplemental Digital Content. Smoking status was defined as current smoking or non-smoking, irrespective of previous smoking status, and race was defined as Black, Hispanic or White (any non-Black or non-Hispanic race). The effect of a dichotomous covariate ' $\mathrm{x}$ ' was modelled as (equation 2):

$$
\theta=\theta_{0} \times\left(1+\theta_{\mathrm{x}} \times \mathrm{x}\right)
$$

where $\theta_{0}$ denotes the population value of the parameter for the null value of the covariate $\mathrm{x}\left(\right.$ i.e. $\mathrm{x}=0$ ). The parameter $\theta_{\mathrm{x}}$ denotes the fractional change in $\theta_{0}$ when $x=1$. Continuous covariates were modelled as multiplicative effects of the form (equation 3 ):

$$
\theta=\theta_{0} \times\left(\mathrm{x} / \mathrm{x}_{\mathrm{norm}}\right)^{\theta_{\mathrm{x}}}
$$

where $\theta_{0}$ denotes the population value of the parameter when $\mathrm{x}=\mathrm{x}_{\text {norm }}\left(\mathrm{e}\right.$.g. $\mathrm{x}_{\text {norm }}=40$ years of age). The parameter $\theta$ denotes 

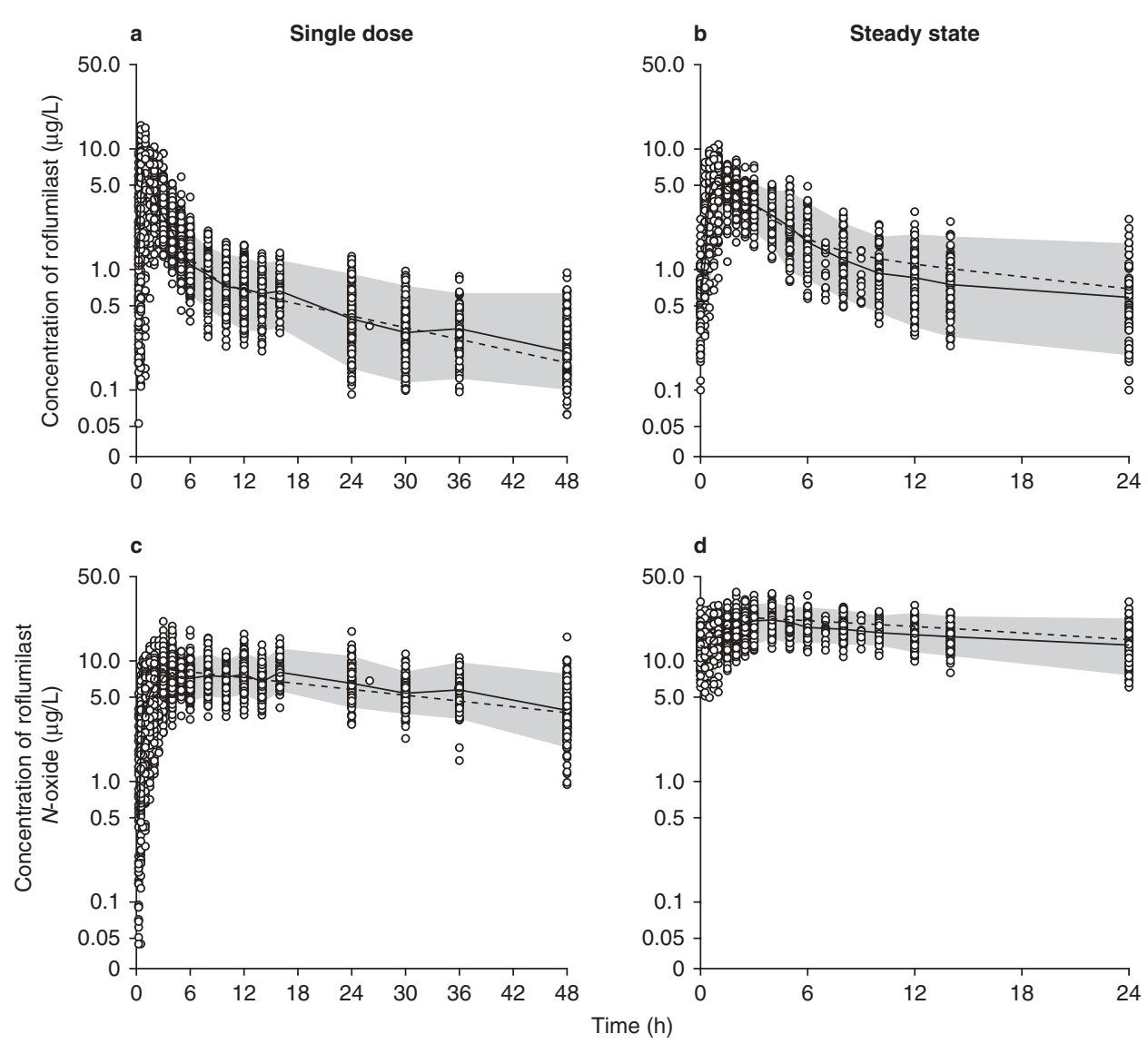

Fig. 1. Concentration-time profiles of roflumilast and roflumilast $N$-oxide in healthy subjects: (a, c) after a single dose; and (b, d) at steady state on day 7 . The data are shown as observations (white circles), median concentration-time profiles (solid lines), population predictions from the base model (dashed lines) and nonparametric 5-95\% ranges (shaded areas).

the population value conditional on the value of $\mathrm{x}$, which is proportional to the power $\theta_{\mathrm{x}}$. When $\theta_{\mathrm{x}}=1, \theta$ is directly proportional to $\mathrm{x}$. The covariates included in the full models for roflumilast and roflumilast $N$-oxide are listed in table I.

\section{Final Model}

For both roflumilast and roflumilast $N$-oxide, the full models were subjected to the Wald approximation method (WAM) to develop final (i.e. more parsimonious) models. ${ }^{[28]}$ Maximization of Schwarz's Bayesian criterion (SBC) was used to rank all possible models, and the top 15 ranked models were then fitted using NONMEM ${ }^{\circledR}$ to calculate the actual likelihood ratio test statistic (LRT $=\Delta$ objective function value $[\mathrm{OFV}]$ ) and $\mathrm{SBC}$. The final parsimonious model was selected, based on the maximum NONMEM ${ }^{\circledR}$-based SBC among these top 15 ranked models.

At all stages of model development (base, full and final), diagnostic plots were examined to assess model adequacy, possible lack of fit or violation of assumptions. Concordance plots of observed values versus population-predicted values and observed values versus individual-predicted values were evaluated for randomness around the line of unity. Plots of weighted residuals (WRES) and individual weighted residuals (IWRES) versus observed values were evaluated for randomness around the zero line. These diagnostic plots were also stratified by dose, regimen (e.g. single dose or steady state) and study to ensure adequacy of the fit across these design factors. Once a stable full model was obtained, box plots of the $\eta \mathrm{s}$ (empirical Bayesian predictions of the interindividual random effects) versus dose and study were also generated to evaluate dose invariance and adequacy of pooling studies for this analysis, respectively. Outliers were identified using both the base and final models, using the criteria $\mid$ WRES $\mid>6$ or $\mid$ IWRES $\mid>6$. The influence of the set of outliers was evaluated by comparing estimates of the key pharmacokinetic parameters with and without the outliers removed. The outliers were considered influential if the parameter estimates differed by more than $15 \%$. After the final model selection, it was decided to test race as a covariate on parent and metabolite clearance and on the relative bioavailability of roflumilast $N$-oxide. 
$\stackrel{\odot}{\sim}$ Table I. Parameter estimates for the roflumilast and roflumilast $N$-oxide models

\begin{tabular}{|c|c|c|c|c|c|c|}
\hline Parameter & Covariate & Base (mean [SE]) & Full (mean [SE]) & $\begin{array}{l}\text { Initial final } \\
\text { (mean [SE]) }\end{array}$ & $\begin{array}{l}\text { Final with race } \\
\text { (mean }[S E] \text { ) }\end{array}$ & $\begin{array}{l}\text { Final for COPD } \\
\text { patients (mean [SE]) }\end{array}$ \\
\hline \multicolumn{7}{|l|}{ Roflumilast model } \\
\hline \multicolumn{7}{|l|}{ OFV (points) } \\
\hline total & & -9023.112 & -9391.524 & -9383.408 & -9397.811 & 272.091 \\
\hline drop from base & & NA & 368.412 & 360.296 & 374.699 & NA \\
\hline nearness to full & & -368.412 & NA & 8.116 & NA & NA \\
\hline \multirow[t]{2}{*}{$\theta_{1}: t_{\text {lag }}(h)$} & No & $0.152[0.0159]$ & $0.158[0.0149]$ & $0.159[0.0148]$ & $0.158[0.0150]$ & $0.158[\mathrm{NA}]$ \\
\hline & $\theta_{9}:$ Food & NA & $-0.307[0.294]$ & $-0.308[0.294]$ & $-0.308[0.296]$ & $-0.308[\mathrm{NA}]$ \\
\hline \multirow[t]{2}{*}{$\theta_{2}: k_{a}\left(h^{-1}\right)$} & No & $0.493[0.0223]$ & $0.533[0.0293]$ & $0.532[0.0285]$ & $0.533[0.0290]$ & $0.533[\mathrm{NA}]$ \\
\hline & $\theta_{11}:$ Food & NA & $-0.701[0.155]$ & $-0.699[0.152]$ & $-0.699[0.153]$ & $-0.699[\mathrm{NA}]$ \\
\hline \multirow[t]{7}{*}{$\theta_{3}: \mathrm{CL} / \mathrm{F}(\mathrm{L} / \mathrm{h})$} & No & $12.7[0.294]$ & $9.92[0.446]$ & $9.93[0.433]$ & $10.5[0.490]$ & $10.5[\mathrm{NA}]$ \\
\hline & $\theta_{12}:$ Age $(y)$ & NA & $-0.140[0.0550]$ & NA & NA & NA \\
\hline & $\theta_{13}:$ Sex $=$ male & NA & $0.203[0.0603]$ & $0.221[0.0602]$ & $0.191[0.0588]$ & $0.191[\mathrm{NA}]$ \\
\hline & $\theta_{15}:$ Smoking & NA & $0.344[0.0588]$ & $0.356[0.0594]$ & $0.307[0.0584]$ & $0.307[\mathrm{NA}]$ \\
\hline & $\theta_{20}:$ Race $=$ Black & NA & NA & NA & $-0.140[0.0713]$ & $-0.140[\mathrm{NA}]$ \\
\hline & $\theta_{21}:$ Race $=$ Hispanic & NA & NA & NA & $-0.297[0.0581]$ & $-0.297[\mathrm{NA}]$ \\
\hline & $\theta_{22}:$ COPD & NA & NA & NA & NA & $-0.394[0.0230]$ \\
\hline \multirow[t]{3}{*}{$\theta_{4}: V_{1}(L)$} & No & $13.1[1.07]$ & $13.9[1.38]$ & $14.3[1.33]$ & $14.3[1.37]$ & $14.3[\mathrm{NA}]$ \\
\hline & $\theta_{17}:$ Bodyweight $(\mathrm{kg})$ & NA & $0.497[0.161]$ & NA & NA & NA \\
\hline & $\theta_{25}:$ COPD & NA & NA & NA & NA & $1.84[0.328]$ \\
\hline$\theta_{5}: Q(\mathrm{~L} / \mathrm{h})$ & No & $20.1[0.487]$ & $20.3[0.517]$ & $20.3[0.507]$ & $20.3[0.514]$ & 20.3 [NA] \\
\hline$\theta_{6}: V_{2}(L)$ & No & $204[5.08]$ & $201[5.17]$ & $201[5.14]$ & $201[5.15]$ & $201[N A]$ \\
\hline \multirow[t]{2}{*}{$F_{\text {rel }}$} & NA & 1 & 1 & 1 & 1 & 1 \\
\hline & $\theta_{19}:$ Food & NA & $0.0214[0.0720]$ & NA & NA & NA \\
\hline$\theta_{7}: \mathrm{CV}\{\sigma\}(\%)$ & & $25.9[0.956]$ & $25.1[0.756]$ & $25.1[0.755]$ & $25.1[0.756]$ & $54.5[0.0392]$ \\
\hline$\omega^{2}\left(\eta\left\{\mathrm{t}_{\text {lag }}\right\}\right)$ & & $2.29[1.79]$ & $1.73[1.35]$ & $1.72[1.34]$ & $1.73[1.36]$ & $1.73[\mathrm{NA}]$ \\
\hline$\omega^{2}\left(\eta\left\{k_{a}\right\}\right)$ & & $0.114[0.0285]$ & $0.155[0.0761]$ & $0.154[0.0713]$ & $0.154[0.0730]$ & $0.154[\mathrm{NA}]$ \\
\hline$\omega^{2}(\eta\{\mathrm{CL} / \mathrm{F}\})$ & & $0.172[0.0136]$ & $0.140[0.0109]$ & $0.142[0.0113]$ & $0.136[0.0111]$ & $0.136[\mathrm{NA}]$ \\
\hline$\omega^{2}\left(\eta\left\{\mathrm{V}_{1}\right\}\right)$ & & $0.713[0.0804]$ & $0.729[0.0765]$ & $0.734[0.0758]$ & $0.734[0.0776]$ & $0.734[\mathrm{NA}]$ \\
\hline$\omega^{2}(\eta\{Q\})$ & & $0.0740[0.0115]$ & $0.0726[0.0107]$ & $0.0727[0.0106]$ & $0.0726[0.0106]$ & $0.0726[\mathrm{NA}]$ \\
\hline$\omega^{2}\left(\eta\left\{V_{2}\right\}\right)$ & & $0.115[0.0131]$ & $0.117[0.0127]$ & $0.118[0.0126]$ & $0.117[0.0124]$ & $0.117[\mathrm{NA}]$ \\
\hline$\omega\left(\eta\left\{Q, V_{2}\right\}\right)$ & & $0.0737[0.0111]$ & $0.0701[0.0101]$ & 0.0705 [0.0103] & $0.0703[0.0100]$ & $0.0703[\mathrm{NA}]$ \\
\hline & & & & & & Continued next page \\
\hline
\end{tabular}




\begin{tabular}{|c|c|c|c|c|c|c|}
\hline Parameter & Covariate & Base (mean [SE]) & Full (mean [SE]) & $\begin{array}{l}\text { Initial final } \\
\text { (mean [SE]) }\end{array}$ & $\begin{array}{l}\text { Final with race } \\
\text { (mean [SE]) }\end{array}$ & $\begin{array}{l}\text { Final for COPD } \\
\text { patients (mean [SE]) }\end{array}$ \\
\hline \multicolumn{7}{|c|}{ Roflumilast $\mathrm{N}$-oxide model } \\
\hline \multicolumn{7}{|l|}{ OFV (points) } \\
\hline total & & -10310.430 & -10982.441 & -10974.671 & -11064.271 & -726.056 \\
\hline drop from base & & NA & 672.011 & 664.241 & 753.841 & NA \\
\hline nearness to full & & -672.011 & NA & 7.770 & NA & NA \\
\hline$\theta_{1}: t_{\text {lag }}(h)$ & No & $0.149[0.0219]$ & $0.157[0.0194]$ & $0.157[0.0195]$ & $0.156[0.0194]$ & $0.156[N A]$ \\
\hline \multirow[t]{2}{*}{$\theta_{2}: D_{1}(h)$} & No & $2.28[0.0754]$ & $2.22[0.0696]$ & $2.22[0.0697]$ & $2.21[0.0700]$ & $2.21[\mathrm{NA}]$ \\
\hline & $\theta_{6}:$ Food & NA & $2.41[0.416]$ & $2.37[0.354]$ & $2.36[0.352]$ & $2.36[N A]$ \\
\hline \multirow[t]{7}{*}{$\theta_{3}: \mathrm{CL} / \mathrm{F}(\mathrm{L} / \mathrm{h})$} & No & $1.05[0.0283]$ & $0.773[0.0468]$ & $0.814[0.0445]$ & $0.883[0.0472]$ & $0.883[\mathrm{NA}]$ \\
\hline & $\theta_{7}:$ Age $(y)$ & NA & $-0.480[0.0981]$ & $-0.486[0.179]$ & $-0.471[0.106]$ & $-0.471[\mathrm{NA}]$ \\
\hline & $\theta_{8}:$ Sex $=$ male & NA & $0.567[0.126]$ & $0.418[0.107]$ & $0.467[0.108]$ & $0.467[\mathrm{NA}]$ \\
\hline & $\theta_{9}:$ Bodyweight $(\mathrm{kg})$ & NA & $-0.497[0.185]$ & NA & NA & NA \\
\hline & $\theta_{10}:$ Smoking & NA & $0.308[0.0758]$ & $0.314[0.0646]$ & $0.235[0.0590]$ & $0.235[\mathrm{NA}]$ \\
\hline & $\theta_{11}:$ Alcohol & NA & $0.00354[0.0626]$ & NA & NA & NA \\
\hline & $\theta_{25}:$ COPD & NA & NA & NA & NA & $-0.0785[0.0324]$ \\
\hline \multirow[t]{3}{*}{$\theta_{4}: V_{d}(L)$} & No & $56.5[1.03]$ & 60.3 [2.33] & $60.8[2.14]$ & $65.8[1.94]$ & $65.8[\mathrm{NA}]$ \\
\hline & $\theta_{12}:$ Bodyweight $(\mathrm{kg})$ & NA & $0.824[0.142]$ & $0.818[0.144]$ & $1.00[0.117]$ & $1.00[\mathrm{NA}]$ \\
\hline & $\theta_{26}:$ COPD & NA & NA & NA & NA & $-0.214[0.0706]$ \\
\hline \multirow[t]{8}{*}{$\mathrm{F}_{\text {rel }}$} & NA & 1 & 1 & 1 & 1 & 1 \\
\hline & $\theta_{13}:$ Age $(y)$ & NA & $-0.315[0.0395]$ & $-0.316[0.0682]$ & $-0.269[0.0404]$ & -0.269 [NA] \\
\hline & $\theta_{14}:$ Sex $=$ male & NA & $0.162[0.0562]$ & $0.154[0.0517]$ & $0.231[0.0465]$ & $0.231[\mathrm{NA}]$ \\
\hline & $\theta_{16}:$ Smoking & NA & 0.0129 [0.0335] & NA & NA & NA \\
\hline & $\theta_{17}:$ Alcohol & NA & $-0.0239[0.0338]$ & NA & NA & NA \\
\hline & $\theta_{18}:$ Food & NA & $0.0133[0.0347]$ & NA & NA & NA \\
\hline & $\theta_{21}:$ Race $=$ Black & NA & NA & NA & $0.431[0.0525]$ & $0.431[\mathrm{NA}]$ \\
\hline & $\theta_{22}:$ Race = Hispanic & NA & NA & NA & $0.267[0.0751]$ & $0.267[\mathrm{NA}]$ \\
\hline$\theta_{5}: \mathrm{CV}\{\sigma\}(\%)$ & & $25.1[0.830]$ & $24.0[0.728]$ & $24.0[0.729]$ & $24.1[0.727]$ & 20.9 [0.0140] \\
\hline$\omega^{2}\left(\eta\left\{D_{1}\right\}\right)$ & & $0.254[0.0240]$ & $0.262[0.0245]$ & $0.262[0.0247]$ & $0.268[0.0245]$ & $0.268[\mathrm{NA}]$ \\
\hline$\omega^{2}(\eta\{C L / F\})$ & & $0.194[0.0247]$ & 0.159 [0.0209] & $0.164[0.0212]$ & $0.150[0.0206]$ & $0.150[\mathrm{NA}]$ \\
\hline$\omega^{2}\left(\eta\left\{V_{d}\right\}\right)$ & & $0.0814[0.00748]$ & $0.0523[0.00524]$ & $0.0526[0.00511]$ & 0.0449 [0.00495] & $0.0449[\mathrm{NA}]$ \\
\hline$\omega\left(\eta\left\{D_{1}, C L / F\right\}\right)$ & & $-0.00800[0.0128]$ & $0.00843[0.00421]$ & $0.0113[0.00563]$ & 0.0221 [0.00688] & $0.0221[\mathrm{NA}]$ \\
\hline$\omega\left(\eta\left\{D_{1}, V_{d}\right\}\right)$ & & $0.0505[0.00930]$ & $0.0377[0.0103]$ & $0.0385[0.00811]$ & 0.0536 [0.00885] & $0.0536[\mathrm{NA}]$ \\
\hline$\omega\left(\eta\left\{C L / F, V_{d}\right\}\right)$ & & $-0.0200[0.00831]$ & $-0.000532[0.00766]$ & $-0.000475[0.00394]$ & $-0.0110[0.00502]$ & $-0.0110[\mathrm{NA}]$ \\
\hline
\end{tabular}

$\boldsymbol{\eta}=$ random effect; $\boldsymbol{\theta}=$ parameter of interest; $\boldsymbol{\sigma}=$ intra-individual variability; $\boldsymbol{\omega}=$ interindividual variability; $\boldsymbol{\omega}^{2}=$ variance; $\mathbf{C L} / \mathbf{F}=$ apparent oral clearance; $\mathbf{C O P D}=$ chronic obstructive pulmonary disease; $\mathbf{C V}=$ coefficient of variation; $\mathbf{D}_{\mathbf{1}}=$ duration of formation; $\mathbf{F}_{\text {rel }}=$ bioavailability of roflumilast $N$-oxide relative to that of roflumilast; $\mathbf{k}_{\mathbf{a}}=$ apparent first-order absorption rate constant; $\mathbf{N A}=$ not applicable; $\mathbf{O F V}=$ objective function value; $\mathbf{Q}=$ apparent intercompartmental clearance; $\mathbf{S E}=$ standard error; $\mathbf{t}_{\mathbf{l a g}}=$ lag time parameter; $\mathbf{V}_{\mathbf{d}}=$ apparent volume of distribution;

$\mathbf{V}_{\mathbf{1}}=$ apparent central volume of distribution; $\mathbf{V}_{\mathbf{2}}=$ apparent peripheral volume of distribution. 


\section{Extension to Chronic Obstructive Pulmonary Disease (COPD) Patients}

The phase I model was extended using data from phase II (IN-108) and phase III $^{[29]}$ studies to estimate the differences between healthy subjects and patients with COPD. (Key inclusion and exclusion criteria, sample retrieval timings and demographic characteristics are shown in tables S- $4 a, S-4 b, S-4 c$ and S-4d, respectively, in the Supplemental Digital Content.)

The analysis of roflumilast used 771 observations from 228 subjects; the roflumilast $N$-oxide analysis was based on 703 observations from 208 subjects. A fixed-effect $\theta_{\text {COPD }}$ was tested by multiplying each pharmacokinetic parameter, in turn, by $\left(1+\mathrm{COPD} \times \theta_{\mathrm{COPD}}\right)$. Since the phase I dataset contained more information, we did not re-estimate the previously found covariates. The only non-fixed parameters in each NONMEM ${ }^{\circledR}$ run were the $\theta_{\text {COPD }}$ parameter and residual error. A forward inclusion was performed after all pharmacokinetic parameters were identified, for which the NONMEM $^{\circledR}$ run including a $\theta_{\text {COPD }}$ on that parameter converged, the $\mathrm{OFV}$ dropped by at least 3.84 points so that the LRT indicated a significant $(\mathrm{p}<0.05)$ COPD dependency and the $95 \%$ confidence interval (CI) of the $\theta_{\mathrm{COPD}}$ did not contain 0 . A backward deletion step was not needed, as there were never more than two pharmacokinetic parameters with a $\theta_{\mathrm{COPD}}$ added.

\section{Verification of the Population Models}

The predictive performances of the final roflumilast and roflumilast $N$-oxide models with race and the final models for COPD patients were compared with noncompartmental estimates of the area under the plasma concentration-time curve (AUC) from 0 to 24 hours at steady state $\left(\mathrm{AUC}_{24}\right)$ - i.e. only multiple-dose data were used. For validation purposes, phase I data were split into index and validation datasets. This provided an additional and more robust assessment of the predictive performance of the model for those covariates that were adequately represented in the validation dataset. Because of the limited number of data from COPD patients, all data were used for model building and an internal validation was performed. For the final model, box plots of the noncompartmental AUCs that were dose normalized to $500 \mu \mathrm{g}$ were constructed to illustrate the consistency between the data-derived (noncompartmental) and model-based estimates of the covariate effects on the extent of exposure (i.e. the $\mathrm{CL}$ or $\mathrm{F}_{\text {rel }}$ ). The noncompartmental $\mathrm{AUC}_{24}$ estimates were also plotted against the corresponding population predictions to assess the degree of concordance. The geometric mean percentage deviations of the noncompartmental AUCs from the model-based predicted AUCs were tabulated by covariate.
Total Phosphodiesterase-4 Inhibitory Activity (TPDE4i) Model

Pharmacokinetic parameter estimates from the population pharmacokinetic models were used to obtain TPDE4i estimates for individuals and the population. tPDE4i is a composite parameter of roflumilast and roflumilast $N$-oxide exposure. It accounts for the intrinsic activity (the drug concentration producing $50 \%$ inhibition $\left[\mathrm{IC}_{50}\right]$ ), protein binding (the unbound fraction $\left[\mathrm{f}_{\mathrm{u}}\right]$ ), and in vivo exposure (the AUC) of both moieties (equation 4):[19]

$$
\text { tPDE4i }=\frac{\mathrm{AUC}_{\mathrm{p}} \times \mathrm{f}_{\mathrm{u}, \mathrm{p}}}{\mathrm{IC}_{50, \mathrm{p}} \times \tau}+\frac{\mathrm{AUC}_{\mathrm{m}} \times \mathrm{f}_{\mathrm{u}, \mathrm{m}}}{\mathrm{IC}_{50, \mathrm{~m}} \times \tau}
$$

where $\mathrm{AUC}_{\mathrm{p}}$ is the AUC of roflumilast $(\mu \mathrm{g} \bullet \mathrm{h} / \mathrm{L}$; either the AUC from time zero to infinity $\left[\mathrm{AUC}_{\infty}\right]$ following a single dose or the AUC over a dosing interval $\left[\mathrm{AUC}_{\tau}\right]$ following multiple doses at steady state); $\mathrm{AUC}_{\mathrm{m}}$ is the AUC of roflumilast $N$-oxide $(\mu \mathrm{g} \bullet \mathrm{h} / \mathrm{L})$; $\mathrm{f}_{\mathrm{u}, \mathrm{p}}$ is the $\mathrm{f}_{\mathrm{u}}$ of roflumilast (\%) measured in vitro; $\mathrm{f}_{\mathrm{u}, \mathrm{m}}$ is the $\mathrm{f}_{\mathrm{u}}$ of roflumilast $N$-oxide (\%) measured in vitro; $\mathrm{IC}_{50, \mathrm{p}}$ is the roflumilast concentration resulting in 50\% PDE4 inhibition in vitro $(\mu \mathrm{g} / \mathrm{L})$; $\mathrm{IC}_{50, \mathrm{~m}}$ is the roflumilast $N$-oxide concentration resulting in $50 \%$ PDE4 inhibition in vitro $(\mu \mathrm{g} / \mathrm{L})$; and $\tau$ is the dosing interval (24 hours for once-daily dosing).

\section{Analysis of Robustness}

A robustness analysis was performed to evaluate the stability and robustness of parameter estimates under the variability of the population. This allowed us to evaluate the usefulness of the model in predicting exposure levels and degrees of confidence within the predicted parameters and covariate effects. Robustness analyses were performed on all intercept terms and covariate effects in the population pharmacokinetic models for roflumilast and roflumilast $N$-oxide by using bootstrapping (resampling) techniques. From the data (healthy subjects and COPD patients) that were used, more than 300 resampled datasets were created at the individual subject level (all observations per subject were grouped in the resampling process). NONMEM ${ }^{\circledR}$ was used to fit the final models to the resampled datasets. For each parameter i, the distribution of the $\mathrm{N}$ bootstrap estimates $\theta_{i}^{(1 \ldots \mathrm{N})}$ was compared with the parameter estimate $\theta_{i}^{*}$, obtained from the original dataset, and its standard error $\mathrm{SE}_{\mathrm{i}}^{*}$, as reported by NONMEM ${ }^{\circledR}$.

The robustness of the parameter estimates was checked using the following procedures:

1. The shapes of the normal approximation $\mathrm{N}\left(\theta_{\mathrm{i}}^{*}, \mathrm{SE}_{\mathrm{i}}^{*}\right)$ and the bootstrap distribution of the $\theta_{i}^{(k)}(\mathrm{k}=1$...N) were compared by means of their first three moments, i.e. mean, variance and skewness. ${ }^{[30]}$

2. Bias-corrected bootstrap 95\% CIs were computed according to the method of Efron and Tibshirani ${ }^{[31]}$ and checked to confirm whether they contained 0 . 
3. The number of cases where a bootstrap estimate $\theta_{i}{ }^{(k)}$ had the opposite algebraic sign to $\theta_{i}^{*}$ was counted (for covariate effects only).

4. For each parameter $\mathrm{i}$, all bootstrap estimates $\theta_{i}^{(\mathrm{k})}$ were tested to check whether they had the same algebraic sign (positive or negative) as $\theta_{i}^{*}$. The test was based on the assumed normal distribution of $\theta_{i}^{(\mathrm{k})} \sim \mathrm{N}\left(\theta_{\mathrm{i}}^{(\mathrm{k})}, \mathrm{SE}_{\mathrm{i}}^{(\mathrm{k})}\right)$, where $\mathrm{SE}_{\mathrm{i}}{ }^{(\mathrm{k})}$ is the standard error of $\theta_{i}^{(k)}$ calculated by NONMEM ${ }^{\circledR}$. The algorithm has previously been explained in detail. ${ }^{[30]}$ The resulting p-values were computed, and the fraction of p-values less than 0.05 is reported (for covariate effects only).

\section{Posterior Predictive Checks for the Areas Under the Plasma Concentration-Time Curves of Roflumilast and Roflumilast $\mathrm{N}$-Oxide and for PPDE4i}

For all subjects used during the model development (phases I-III), 500 simulations for the $\mathrm{AUC}_{\mathrm{p}}, \mathrm{AUC}_{\mathrm{m}}$ and $\mathrm{tPDE} 4 \mathrm{i}$ were computed. These predicted values were compared with AUCs and tPDE4i values obtained by noncompartmental evaluation of observed concentrations by locating each value relative to the distribution of its 500 predictions, similar to the concept of standardized visual predictive checks. ${ }^{[32]}$ In particular, the number of observations within the $95 \%$ CI of its 500 predictions was evaluated.

Population predictions from the parameter models were computed using NONMEM $^{\circledR}$. The geometric mean and the parametric 95\% CI were calculated (assuming a log-normal distribution) for each set of simulated values. These CIs were then projected (shifted and scaled) to the interval $[-1,1]$, which automatically projects the geometric mean to 0 . The noncompartmental analysis (NCA) AUCs were subsequently transformed using the same projections as were obtained from their corresponding predictions. These transformed NCA AUCs were grouped by various subpopulations and represented as histograms for visualization and numerical analysis.

\section{Adverse Event (AE) Model}

The analyses were performed on the phase III, double-blind, randomized, placebo-controlled study (M2-110). ${ }^{[29]}$ Modelling of AEs was performed using data from 909 patients with moderate-to-severe COPD, who were randomly assigned to receive roflumilast $500 \mu \mathrm{g}$ or placebo given orally once daily for 24 weeks. Samples for pharmacokinetic analysis were taken from a subset of all patients. Study details and demographic characteristics are shown in table S-4 in the Supplemental Digital Content. AEs were recorded using standard International Conference on Harmonization guidelines for good clinical practice, coded according to the Medical Dictionary for Regulatory Activities ${ }^{[33]}$ and analysed on the preferred term (PT) level. Determination of the severity and causality of AEs was left to the investigator's discretion.

In total, 1938 AEs from 453 different PT categories were reported in the treatment phase of this study. In order to ensure sufficient data to fit the logistic regression models, only prominent AES from different PT categories occurring in more than $2 \%$ of all patients (i.e. 19 or more patients) were analysed (see table S-5 in the Supplemental Digital Content). A two-step approach was followed in developing the AE model for each PT category. First, the occurrence of AEs within the given PT category was tested on treatment dependence. A variable $\mathrm{AE}$ was assigned to every subject: $\mathrm{AE}=1$ if the subject had at least one $\mathrm{AE}$ in the given PT category, and $\mathrm{AE}=0$ if otherwise. Since no specific distribution of $\mathrm{AE}$ was assumed, a permutation test was used to test the hypothesis (equation 5):

$$
\mathrm{H}_{1}: \mathrm{P}(\mathrm{AE}=1 \mid \text { dose }=500)>\mathrm{P}(\mathrm{AE}=1 \mid \text { dose }=0)
$$

In order not to lose any PT category that might later reveal an exposure dependency, a very conservative $\mathrm{p}$-value threshold of 0.2 was used.

The second step for all PTs with $\mathrm{p}$-values of $<0.2$ was to fit a logistic regression model to use exposure to predict the probability of AEs (approximated by the frequency of AEs among the subjects in the dataset) on the subset of treated subjects. For each PT passing with a $p$-value of $<0.2$, three models were set up - one model for each of the following exposure variables: $\mathrm{AUC}_{\mathrm{p}}, \mathrm{AUC}_{\mathrm{m}}$ and tPDE4i. AUC $\mathrm{p}$ and $\mathrm{AUC}_{\mathrm{m}}$ values were determined using data from blood samples and were used to estimate tPDE4i for each patient. In total, 177 patients treated with roflumilast had pharmacokinetic samples, and data from these patients were used to form the AE models.

The capability of the models to predict the probability of AEs was evaluated for each explanatory variable. logit(AE) was used as the response variable of the model. The significance of each model parameter was tested using an LRT between the models, with and without this parameter. If the $\mathrm{p}$-value for exposure was $<0.05$ (i.e. exposure was a significant predictor of the probability of developing an AE), the covariates age, sex, race, bodyweight and height were additionally tested by a full forward inclusion/backward deletion strategy. A nonparametric bootstrap procedure was used to estimate the $95 \%$ CI of the mean model prediction.

\section{Model Validation}

The logistic models describe the probability of a subject developing an AE of the PT category under investigation, based 
on exposure to the roflumilast parent and metabolite. In order to compare this prediction with the observed AEs, a threshold $\alpha$ was used to map the predicted probabilities to the two cases: 'AE' if $\mathrm{p}(\mathrm{AE})>\alpha$, or 'no $\mathrm{AE}$ ' if $\mathrm{p}(\mathrm{AE}) \leq \alpha$. The concordance of predictions and observations was counted to validate the fitted models. Specificity (Sp) and sensitivity ( $\mathrm{Sn})$ were calculated as functions of this threshold.

The area under the receiver operating characteristic (ROC) curve $^{[34]}\left(\mathrm{AUC}_{\mathrm{ROC}}\right)$ and the maximum weighted accuracy $\left(\mathrm{Acc}_{\mathrm{w}, \text { max }}\right)$ were used to describe the predictive performance of the models. A value of 0.5 for the $\mathrm{AUC}_{\mathrm{ROC}}$ would result from 'pure guessing', while an optimal predictive capacity would give $\mathrm{AUC}_{\mathrm{ROC}}=1$. Accuracy is defined as the percentage of correct predictions for a given threshold $\alpha$ : $\operatorname{Acc}(\alpha)=\operatorname{Sn}(\alpha)+\operatorname{Sp}(\alpha)$. Maximum accuracy is defined as $\operatorname{Max}_{\alpha \varepsilon[0.1]}\{\operatorname{Acc}(\alpha)\}$. The concept of weighted accuracy corrects this term in unbalanced cases where the frequencies of subjects with and without AEs are considerably different.

\section{Results}

\section{Roflumilast}

The parameter estimates for the base, full and final models are shown in table I. The diagnostic plots showed no appreciable systematic trends versus time, dose or study (see figure S-1 in the Supplemental Digital Content).

\section{Final Model for Healthy Subjects}

The final parsimonious model included covariate effects for food on the $\mathrm{t}_{\mathrm{lag}}\left(\theta_{9}\right)$ and $\mathrm{k}_{\mathrm{a}}\left(\theta_{11}\right)$ and for sex $\left(\theta_{13}\right)$ and smoking status $\left(\theta_{15}\right)$ on CL. The rank correlation between the WAMand NONMEM ${ }^{\circledR}$-based SBCs was poor $\left(\rho_{\text {Rank }}=0.364\right)$; however, the final model $\left(\right.$ NONMEM $^{\circledR}$ rank $=1$, WAM rank $=9$ ) appeared to be parsimonious, accounting for all but 8.116 points of the 368.412-point decrease in the OFV between the full and base models with three of the seven covariate parameters restricted to 0 (i.e. $\theta_{12}=\theta_{17}=\theta_{19}=0$ ).

When race was included as a covariate on CL in the final model, an additional 14.403-point drop in the OFV was obtained. The final model with race was obtained using the final model for COPD patients given in the next section, setting the COPD value to 0 .

Diagnostic plots for the resulting final model showed no appreciable systematic trends versus time, dose or study. A comparison of the plots of $\eta \mathrm{s}$ versus each covariate for each pharmacokinetic parameter between the base and final models suggested that the covariate effects were adequately described by the final model, as covariate trends observed in the base model were diminished for the final model.

\section{Predictive Performance of the Final Model for Healthy Subjects}

The final model predictions were fairly concordant with the noncompartmental estimates over the range of doses $(250-1000 \mu \mathrm{g})$ that were studied (see table S-6a in the Supplemental Digital Content).

The geometric mean percentage deviations of the dosenormalized observed AUCs from the predicted AUCs were no greater than $6.57 \%$ in the index dataset, supporting the adequacy of the fit of the model (see table S-6a in the Supplemental Digital Content). The validation dataset contained information over a more restricted range. Nevertheless, the population predictions on this independent validation dataset, not used in the development of the model, were in good concordance with the noncompartmental estimates, providing independent information on the predictive performance of the final model. For the validation dataset, the mean percentage deviations were larger than for the index dataset; however, for the bulk of the data (57 observations in non-smoking males), the mean percentage deviation $(7.25 \%)$ was similar to that obtained from the index dataset.

\section{Final Model for COPD Patients}

The final population pharmacokinetic model for roflumilast in patients with COPD was given by equation 6 :

$$
\begin{aligned}
\mathrm{t}_{\text {lag }, \mathrm{i}}= & \theta_{1} \times\left(1+\theta_{9} \times \text { Food }_{\mathrm{i}}\right) \times \mathrm{e}^{\eta\left(\mathrm{t}_{\text {lag }}\right)_{\mathrm{i}}} \\
\mathrm{k}_{\mathrm{a}, \mathrm{i}}= & \theta_{2} \times\left(1+\theta_{11} \times \text { Food }_{\mathrm{i}}\right) \times \mathrm{e}^{\eta\left(\mathrm{k}_{\mathrm{a}}\right)_{\mathrm{i}}} \\
\mathrm{CL}_{\mathrm{i}}= & \theta_{3} \times\left(1+\theta_{13} \times \text { Sex }_{\mathrm{i}}\right) \times\left(1+\theta_{15} \times \text { Smoking }_{\mathrm{i}}\right) \\
& \times\left(1+\theta_{20} \times \text { Race }_{\text {Black, }, \mathrm{i}}\right) \times\left(1+\theta_{21} \times \text { Race }_{\text {Hispanic, } \mathrm{i}}\right) \\
& \times\left(1+\theta_{22} \times \operatorname{COPD}_{\mathrm{i}}\right) \times \mathrm{e}^{\eta(\mathrm{CL})_{\mathrm{i}}} \\
\mathrm{V}_{1, \mathrm{i}}= & \theta_{4} \times\left(1+\theta_{25} \times \operatorname{COPD}_{\mathrm{i}}\right) \times \mathrm{e}^{\eta\left(\mathrm{V}_{1}\right)_{\mathrm{i}}} \\
\mathrm{Q}_{\mathrm{i}}= & \theta_{5} \times \mathrm{e}^{\eta(\mathrm{Q})_{\mathrm{i}}} \\
\mathrm{V}_{2, \mathrm{i}}= & \theta_{6} \times \mathrm{e}^{\eta\left(\mathrm{V}_{2}\right)_{\mathrm{i}}}
\end{aligned}
$$

The estimated covariate effects of COPD indicated that CL was reduced by about $40 \%\left(\theta_{22}=-0.394\right)$ and the $\mathrm{V}_{1}$ was $184 \%$ larger $\left(\theta_{25}=1.84\right)$ in patients with COPD than in healthy subjects (table I).

\section{Predictive Performance of the Final Model for COPD Patients}

Predictive performance checks were performed as in the phase I model. Because of the limited data in phase II and III, we did not split the data into index and validation datasets but 
carried out an internal comparison of model-predicted versus noncompartmental AUCs. The results are summarized in table S-6b in the Supplemental Digital Content.

\section{Roflumilast $\mathrm{N}$-Oxide Metabolite}

The parameter estimates for the base, full and final models are shown in table I. The diagnostic plots showed no appreciable systematic trends versus time, dose or study (see figure S- 1 in the Supplemental Digital Content).

\section{Final Model for Healthy Subjects}

The full model was used with the WAM to determine a parsimonious final model. The high rank correlation between the WAM- and NONMEM ${ }^{\circledR}$-based SBCs $\left(\rho_{\text {Rank }}=0.793\right)$ suggested good performance of the WAM algorithm. The final parsimonious model selected by the WAM, based on the highest conditional ranked NONMEM ${ }^{\circledR}$-based SBC $\left(\right.$ NONMEM $^{\circledR}$ rank $=1$ ), included covariate effects for food on the $D_{1}\left(\theta_{6}\right)$; age $\left(\theta_{7}\right)$, sex $\left(\theta_{8}\right)$ and smoking status $\left(\theta_{10}\right)$ on $C L$; bodyweight on the $\mathrm{V}_{\mathrm{d}}\left(\theta_{12}\right)$; and age $\left(\theta_{13}\right)$ and sex $\left(\theta_{14}\right)$ on the $\mathrm{F}_{\text {rel }}$. This final model was considered parsimonious, as it accounted for all but 7.770 points of the 672.011-point decrease in the OFV between the full and base models with 6 of the 13 covariate parameters restricted to 0 (i.e. $\theta_{9}=\theta_{11}=\theta_{15}=\theta_{16}=\theta_{17}=\theta_{18}=0$ ).

After the development of the final model, the potential effect of race on the $C L$ and $F_{\text {rel }}$ was examined. Race as a covariate on both the $C L$ and $F_{\text {rel }}$ in the final model failed to converge. However, use of race as a covariate on the $\mathrm{F}_{\text {rel }}$ alone resulted in a stable model with an additional 89.6-point drop in the OFV.

Diagnostic plots for the resulting final model showed no appreciable systematic trends versus time, dose or study, suggesting that the final model adequately described the data across these design factors. A comparison of the plots of $\eta$ versus each covariate for each pharmacokinetic parameter between the base and final models suggested that the covariate effects were adequately described by the final model, as covariate trends observed in the base model were diminished for the final model.

The CL of roflumilast $N$-oxide was estimated to decrease by $27.9 \%$ between the ages of 40 and 80 years. When the effects of age on the $C L$ and $F_{\text {rel }}$ were taken into account simultaneously, the AUC increased with age. The effect of age was not shown for the validation dataset, since all subjects were younger than 45 years of age. With regard to sex, males had lower AUCs than females.

\section{Predictive Performance of the Final Model for Healthy Subjects}

The final model predictions were fairly concordant with the noncompartmental estimates over the range of doses studied. The geometric mean percentage deviations of the dosenormalized noncompartmental AUCs from the predicted AUCs (see table S-6a in the Supplemental Digital Content) supported the adequacy of the final model prediction, with the largest absolute mean deviation reported as $-10.1 \%$.

The percentage deviation for male non-smokers was approximately $+17.4 \%$, suggesting poorer predictive performance on the validation dataset than on the index dataset $(-8.7 \%)$. It was expected that the absolute percentage deviations would be larger for the independent validation dataset than for the index dataset.

The largest deviation within the validation dataset was found for female smokers $(\sim 75 \%)$. However, this value was based on only two observations.

\section{Final Model for COPD Patients}

The final population pharmacokinetic model for roflumilast $N$-oxide in patients with COPD is given by (equation 7):

$$
\begin{aligned}
\mathrm{t}_{\text {lag,i }}= & \theta_{1} \times \mathrm{e}^{\eta\left(\mathrm{t}_{\text {lag }}\right)_{\mathrm{i}}} \\
\mathrm{D}_{\mathrm{i}}= & \theta_{2} \times\left(1+\theta_{6} \times \text { Food }_{\mathrm{i}}\right) \times \mathrm{e}^{\eta(\mathrm{D})_{\mathrm{i}}} \\
\mathrm{CL}_{\mathrm{i}}= & \theta_{3} \times\left(\frac{\text { Age }_{\mathrm{i}}}{40}\right)^{\theta_{7}} \times\left(1+\theta_{8} \times \text { Sex }_{\mathrm{i}}\right) \\
& \times\left(1+\theta_{10} \times \text { Smoking }_{\mathrm{i}}\right) \\
& \times\left(1+\theta_{25} \times \mathrm{COPD}_{\mathrm{i}}\right) \times \mathrm{e}^{\eta(\mathrm{CL})_{\mathrm{i}}} \\
\mathrm{V}_{\mathrm{d}, \mathrm{i}}= & \theta_{4} \times\left(\frac{\text { Weight }_{\mathrm{i}}}{70}\right)^{\theta_{12}} \\
& \times\left(1+\theta_{26} \times \mathrm{COPD}_{\mathrm{i}}\right) \times \mathrm{e}^{\eta\left(\mathrm{V}_{\mathrm{d}}\right)_{\mathrm{i}}} \\
\mathrm{F}_{\text {Rel,i }}= & 1 \times\left(\frac{\text { Age }_{\mathrm{i}}}{40}\right)^{\theta_{13}} \times\left(1+\theta_{14} \times \text { Sex }_{\mathrm{i}}\right) \\
& \times\left(1+\theta_{21} \times \text { Race }_{\text {Black }, \mathrm{i}}\right) \\
& \times\left(1+\theta_{22} \times \text { Race }_{\text {Hispanic,i }}\right)
\end{aligned}
$$

The estimated covariate effect of COPD indicated that CL was reduced by $7.8 \%\left(\theta_{25}=-0.0785\right)$ and the $\mathrm{V}_{\mathrm{d}}$ was reduced by $21.4 \%\left(\theta_{26}=-0.214\right)$ in COPD patients (table I). The major differences between healthy subjects and COPD patients were in the $C L$ and $V_{d}$.

\section{Predictive Performance of the Final Model for COPD Patients}

Predictive performance checks were performed as in the phase I model. Because of the limited data in phase II and III, we did not split the data into index and validation datasets but carried out an internal comparison of predicted versus 
NCA-based AUCs. The results are summarized in table S-6b in the Supplemental Digital Content.

\section{Analysis of Robustness}

For most of the model parameters, the normal approximation of parameters provided by NONMEM ${ }^{\circledR}$ was in good agreement with the bootstrap distributions (see table S-7 and figures S-2 to S-5 in the Supplemental Digital Content). However, a few parameters clearly showed different distribution shapes by means of bias, spreads and skewness. This did not per se imply problems with robustness but indicated parameters that should be checked carefully. Table S-7 and figures S-2 to S-5 contain the following information to allow the robustness of the models to be judged: the bias-corrected bootstrap 95\% CIs; the number of cases where a bootstrap estimate $\theta_{i}^{(\mathrm{k})}$ had the opposite algebraic sign as compared with $\theta_{i}^{*}$; and the number of cases where $\theta_{i}^{(k)}$ was significantly $>0$ (if $\theta_{i}^{*}$ was $>0$ ) or $<0$ (if $\theta_{i}^{*}$ was $<0$ ).

The model parameters showed satisfying robustness, with two exceptions ('food on $\mathrm{t}_{\mathrm{lag}}$ ' and 'Race ${ }_{\text {Black }}$ on $\mathrm{CL}$ '). Apart from these two exceptions, no bias-corrected bootstrap 95\% CIs contained 0 , and no more than $2.5 \%$ of all bootstrap estimates had an algebraic sign opposite to those of the original estimates. No parameter had more than $13 \%$ of its bootstrap estimates fail the test on having the same algebraic sign as the original parameter.

'Food on $t_{\text {lag }}$ ' showed severe robustness issues. $t_{\text {lag }}$ could only be fitted using the NONMEM ${ }^{\circledR}$ first-order method, which is well known to give biased estimates. This parameter was neither well described by its NONMEM ${ }^{\circledR}$ normal approximation, nor was it robust after bootstrap evaluation. Its bootstrap CI contained 0 ; only $61 \%$ of the bootstrap parameter estimates were negative, and only $44 \%$ passed the test on being negative (the original parameter estimate was negative). However, since the $t_{\text {lag }}$ does not influence exposure at steady state, this finding was not considered critical for the overall population pharmacokinetic model. 'Race Black $_{\text {on }} \mathrm{CL}$ ' showed minor robustness issues. The bootstrap CI did not contain 0 . However, its upper limit was $-0.0003 ; 36 \%$ of the bootstrap parameter estimates failed the test on being negative, although the original parameter estimate was negative.

\section{Posterior Predictive Checks}

Minor posterior predictive check problems $(10 \%<\%$ values outside the $95 \% \mathrm{CI} \leq 15 \%$ ) were found for middle-aged subjects. The predictive performance of the models for elderly subjects (age $>65$ years) and subjects with a bodyweight $<60 \mathrm{~kg}$, particularly for the roflumilast parent, showed moderate problems $(15 \%<\%$ values outside the $95 \%$ CI $\leq 20 \%)$. However, since approximately $90 \%$ of the tPDE4i is provided by roflumilast
$\mathrm{N}$-oxide, for which only minor issues were seen, the overall finding was not considered critical (see table S-8 and figures S-6 to S-9 in the Supplemental Digital Content).

\section{Covariate Effects on TPDE4i}

A parametric bootstrap estimation method was performed using estimates of roflumilast and roflumilast $N$-oxide exposure from the final population pharmacokinetic models. CIs for relative tPDE4i exposure were computed from 10000 replicates.

The impact of individual covariates was determined from the relative tPDE4i values calculated for Black, Hispanic, female or smoker subgroups of 40 years of age. Of the single covariates, Black race had the greatest impact on tPDE4i (figure 2 and table II). Compared with the reference population (40-year-old, male, non-smoking, White, healthy subjects), tPDE4i was found to be increased by approximately $19.3 \%$ [95\% CI 8.2, 31.6] in women, $28.2 \%$ [95\% CI 4.1, 57.9] in Hispanic subjects, $42.1 \%$ [95\% CI 16.4, 73.4] in Black subjects and 12.6\% [95\% CI -6.6, 35.6] in COPD patients. The tPDE4i increased exponentially with age (e.g. by $8.3 \%$ [95\% CI -11.2, 32.2] in 60-year-olds and by $14.5 \%$ [95\% CI $-9.5,44.7]$ in 80 -year-olds) but was observed to be approximately $19.1 \%$ [95\% CI $0.7,34.0]$ lower in smokers than in non-smokers.

\section{Pharmacokinetic AE Model}

Permutation tests for each prominent PT (AEs in a PT category occurring in $>2 \%$ of patients in the study dataset) produced $p$-values of $<0.2$ for diarrhoea, nausea, headache, dizziness, insomnia, abdominal pain and fatigue (table III). The $p$-values for the estimates of the effect of the $\mathrm{AUC}_{\mathrm{p}}, \mathrm{AUC}_{\mathrm{m}}$ or tPDE4i on these AEs are shown in table IV. Exposure was a significant predictor of the probability of developing diarrhoea, nausea or headache $(\mathrm{p}<0.05)$. No relationship between any exposure measure and insomnia, abdominal pain, dizziness or fatigue could be found. For each exposure variable, the maximum tested model was (equation 8):

$$
\begin{aligned}
\operatorname{logit}\left(\mathrm{AE}_{\mathrm{i}}\right)= & \beta_{0}+\beta_{\text {exposure }} \times \text { exposure }_{\mathrm{i}} \\
& +\beta_{\text {Age }} \times\left(\frac{\text { Age }_{\mathrm{i}}}{40}\right)+\beta_{\mathrm{Sex}} \times \text { Sex }_{\mathrm{i}}+\beta_{\text {Race }} \\
& \times \text { Race }_{\mathrm{i}}+\beta_{\text {Weight }} \times\left(\frac{\text { Weight }_{\mathrm{i}}}{70}\right)+\beta_{\text {Height }} \\
& \times\left(\frac{\text { Height }_{\mathrm{i}}}{170}\right)+\varepsilon_{\mathrm{i}}
\end{aligned}
$$

where $\varepsilon$ is the residual error, $\beta_{0}$ is the intercept, $\beta_{\text {exposure }}$ is the slope of the exposure response and $\beta_{\mathrm{Sex}} \ldots \beta_{\mathrm{Height}}$ are the corresponding covariate effects. 


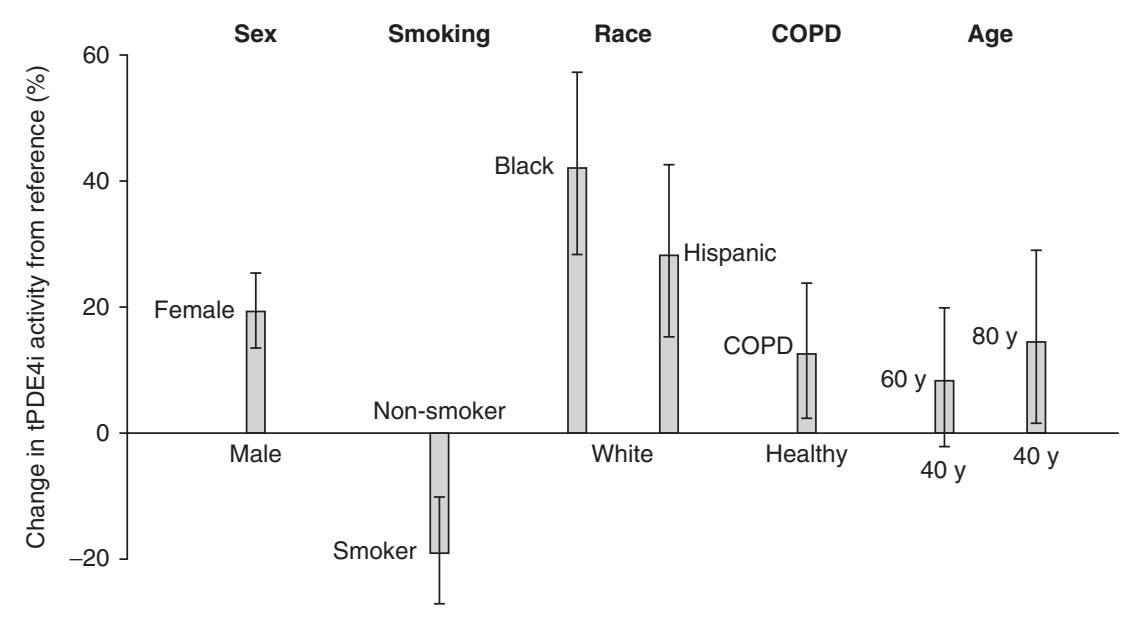

Fig. 2. Estimated covariate effects on total phosphodiesterase-4 inhibitory (tPDE4i) activity relative to the reference population (40-year-old, White, nonsmoking, healthy, male subjects). The data are shown as geometric means and $68 \%$ ranges. COPD $=$ chronic obstructive pulmonary disease.

Model predictions for the probability of developing diarrhoea, nausea or headache are shown in table IV.

The logistic regression model curves for diarrhoea, nausea and headache with tPDE4i as the predictor exposure variable are shown in figure 3 and the ROC plots in figure S-10 in the Supplemental Digital Content. Diarrhoea showed a significant dose and exposure dependency on both the tPDE4i and $\mathrm{AUC}_{\mathrm{m}}$. For example, a subject with a tPDE4i of 1.03 at the population geometric mean would have a $13 \%(95 \%$ CI $7.5,18.5)$ probability of developing diarrhoea. There was no significant covariate. For the tPDE4i logistic model, the $\mathrm{Acc}_{\mathrm{w}, \max }$ was $63.7 \%$ and the $\mathrm{AUC}_{\mathrm{ROC}}$ was 0.63 . A sensitivity analysis showed that a subject at the population's $75 \%$ quantile of exposure $($ tPDE4i $=1.34)$ had a higher probability $(18.2 \%$ compared with $10 \%$ ) of developing diarrhoea than a subject at the $25 \%$ quantile (tPDE4i=0.79).
Nausea also showed a significant dose and exposure dependency on the tPDE4i and $\mathrm{AUC}_{\mathrm{m}}$. The predicted probability of a subject developing nausea (using tPDE4i at the geometric mean) was $6.0 \%$ (95\% CI 2.6, 9.4). There was no significant covariate. The tPDE4i logistic model showed an $\mathrm{Acc}_{\mathrm{w}, \max }$ of $67 \%$ and an $\mathrm{AUC}_{\mathrm{ROC}}$ of 0.72 . A sensitivity analysis showed that a subject at the $75 \%$ quantile of tPDE4i had a higher probability of developing nausea than a subject at the $25 \%$ quantile of tPDE4i (9.5\% vs $4.2 \%)$.

The dose and exposure dependency on $\mathrm{TPDE} 4 \mathrm{i}$ and $\mathrm{AUC}_{\mathrm{m}}$ was also significant for headache. The predicted probability of a subject developing headache with tPDE4i at the geometric mean was $5.1 \%$ (95\% CI 1.9, 8.6). There was no significant covariate. The $\mathrm{Acc}_{\mathrm{w}, \max }$ and $\mathrm{AUC}_{\mathrm{ROC}}$ for the tPDE4i logistic model were $70.3 \%$ and 0.69 , respectively. A sensitivity analysis showed that a subject at the $75 \%$ quantile of tPDE4i had a

Table II. Covariate effects on mean total phosphodiesterase-4 inhibitory activity (tPDE4i): geometric means and 95\% Cls. For every covariate, the relative effect on the mean TPDE4i is listed when switching from the reference population (40-year-old, White, non-smoking, healthy male) to the covariate population

\begin{tabular}{llllc}
\hline Covariate & Reference & & Covariate population & Relative change (\% [95\% Cl]) \\
\hline Sex & Male & $\rightarrow$ & Female & $19.3[8.2,31.6]$ \\
Smoking & Non-smoker & $\rightarrow$ & Smoker & $-19.1[-34.0,-0.7]$ \\
Race & White & $\rightarrow$ & Black & $42.1[16.4,73.4]$ \\
& & & Hispanic & $28.2[4.1,57.9]$ \\
COPD & Healthy & $\rightarrow$ & COPD & $12.6[-6.6,35.6]$ \\
Age (y) & 40 & $\rightarrow$ & 60 & $8.3[-11.2,32.2]$ \\
& & & 80 & $14.5[-9.5,44.7]$ \\
\hline
\end{tabular}


Table III. Results of significance tests for the parameter estimates for each exposure variable

\begin{tabular}{|c|c|c|c|}
\hline \multirow[t]{2}{*}{ Preferred term } & \multicolumn{3}{|l|}{$p$-Value } \\
\hline & $\begin{array}{l}\text { tPDE4i } \\
\text { dependency }\end{array}$ & $\begin{array}{l}\mathrm{AUC}_{\mathrm{p}} \\
\text { dependency }\end{array}$ & $\begin{array}{l}\mathrm{AUC}_{\mathrm{m}} \\
\text { dependency }\end{array}$ \\
\hline Diarrhoea & 0.003 & 0.026 & 0.004 \\
\hline Nausea & 0.003 & 0.017 & 0.003 \\
\hline Headache & 0.018 & 0.191 & 0.017 \\
\hline Insomnia & 0.472 & 0.816 & 0.461 \\
\hline Abdominal pain & 0.661 & 0.585 & 0.674 \\
\hline Dizziness & 0.809 & 0.956 & 0.803 \\
\hline Fatigue & 0.886 & 0.541 & 0.845 \\
\hline
\end{tabular}

$\mathbf{A U C}=$ area under the plasma concentration-time curve; $\mathbf{A U C}_{\mathbf{m}}=\mathrm{AUC}$ of roflumilast $\mathrm{N}$-oxide (metabolite); $\mathbf{A U C}_{\mathbf{p}}=\mathrm{AUC}$ of roflumilast (parent); tPDE4i $=$ total phosphodiesterase-4 inhibitory activity.

higher probability of developing headache than a subject at the $25 \%$ quantile of tPDE4i (7.6\% vs $3.7 \%)$.

\section{Discussion}

A covariate is any variable that is specific to an individual and may influence the pharmacokinetics or pharmacodynamics of a drug in that person. Using a population approach to modelling allows such covariates to be built into the model and thereby allows individualized dosage regimens to be developed. ${ }^{[36]}$
Our work shows that the pharmacokinetics of roflumilast can be modelled adequately using a two-compartment model with first-order absorption including a $t_{\text {lag }}$. Roflumilast clearance was influenced by sex, smoking status and race; the $\mathrm{k}_{\mathrm{a}}$ and $\mathrm{t}_{\text {lag }}$ were influenced by food. The pharmacokinetics of the roflumilast metabolite, roflumilast $N$-oxide, can be modelled adequately using a one-compartment model with zero-order absorption. Roflumilast $N$-oxide clearance was influenced by the subject's age, sex and smoking status; the $F_{\text {rel }}$ was influenced by the subject's age, sex and race; the $\mathrm{V}_{\mathrm{d}}$ was influenced by bodyweight; and the apparent duration of formation was influenced by food.

Our final models for roflumilast and roflumilast $N$-oxide displayed good descriptive and predictive performance. Diagnostic plots for the roflumilast and roflumilast $N$-oxide full models showed no appreciable systematic trends versus time, dose or study. The modelling-based inferences of the effects of the covariates on exposure were supported by data-derived descriptive statistics for the $\mathrm{AUC}_{24}$ at steady state. This was true for the index dataset from which the models were developed and for the independent validation dataset. The predictive performance of both models was comparable to descriptive performance relative to the index dataset. The predictive performance for female smokers was limited by their underrepresentation in the validation dataset $(n=2)$.

Clearance of roflumilast was $19 \%$ greater in men than in women. This finding contradicted those of other studies on drugs that are metabolized via CYPs. ${ }^{[37-39]}$ However, it is likely that the small differences in roflumilast clearance were related

Table IV. Logistic regression model predictions for the probability of developing diarrhoea, nausea or headache

\begin{tabular}{|c|c|c|c|c|c|c|}
\hline$A E$ & tPDE4i & $\begin{array}{l}\text { Predicted probability of } \mathrm{AE} \\
\text { (mean } \%[95 \% \mathrm{Cl}])\end{array}$ & $\mathrm{AUC}_{\mathrm{p}}$ & $\begin{array}{l}\text { Predicted probability of } \\
\mathrm{AE}(\text { mean } \%[95 \% \mathrm{Cl}])\end{array}$ & $\mathrm{AUC}_{\mathrm{m}}$ & $\begin{array}{l}\text { Predicted probability of } \\
\mathrm{AE}(\text { mean } \%[95 \% \mathrm{CI}])\end{array}$ \\
\hline \multicolumn{7}{|l|}{ Diarrhoea } \\
\hline $2.5 \%$ quantile & 0.41 & $6.4[2.5,13.0]$ & 30.5 & $8.0[3.1,16.1]$ & 208 & $6.6[2.3,13.5]$ \\
\hline Geometric mean & 1.03 & $13.0[7.5,18.5]$ & 60.2 & $14.1[8.9,19.4]$ & 523 & $12.9[7.1,18.0]$ \\
\hline $97.5 \%$ quantile & 2.15 & $38.3[19.3,57.7]$ & 110.6 & $32.5[14.1,54.3]$ & 1144 & $39.0[18.4,58.1]$ \\
\hline \multicolumn{7}{|l|}{ Nausea } \\
\hline $2.5 \%$ quantile & 0.41 & $2.3[0.6,5.5]$ & 30.5 & $2.9[0.7,6.9]$ & 208 & $2.4[0.5,5.7]$ \\
\hline Geometric mean & 1.03 & $6.0[2.6,9.4]$ & 60.2 & $6.7[3.1,10.7]$ & 523 & $5.9[2.5,9.8]$ \\
\hline $97.5 \%$ quantile & 2.15 & $27.5[11.4,49.0]$ & 110.6 & $23.8[8.9,46.1]$ & 1144 & $28.1[10.5,50.9]$ \\
\hline \multicolumn{7}{|l|}{ Headache } \\
\hline $2.5 \%$ quantile & 0.41 & $2.3[0.5,6.0]$ & 30.5 & $3.7[0.9,9.5]$ & 208 & $2.3[0.5,5.5]$ \\
\hline Geometric mean & 1.03 & $5.1[1.9,8.6]$ & 60.2 & $6.1[2.7,10.0]$ & 523 & $5.0[1.8,8.3]$ \\
\hline $97.5 \%$ quantile & 2.15 & $19.8[5.9,37.4]$ & 110.6 & $13.6[2.9,30.0]$ & 1144 & $20.4[6.7,41.0]$ \\
\hline
\end{tabular}

$\mathbf{A E}=$ adverse event; $\mathbf{A U C}=$ area under the plasma concentration-time curve; $\mathbf{A U C}_{\mathbf{m}}=\mathrm{AUC}$ of roflumilast $\mathrm{N}$-oxide (metabolite); $\mathbf{A U C} \mathbf{C}_{\mathbf{p}}=\mathrm{AUC}$ of roflumilast (parent); tPDE4i = total phosphodiesterase-4 inhibitory activity. 

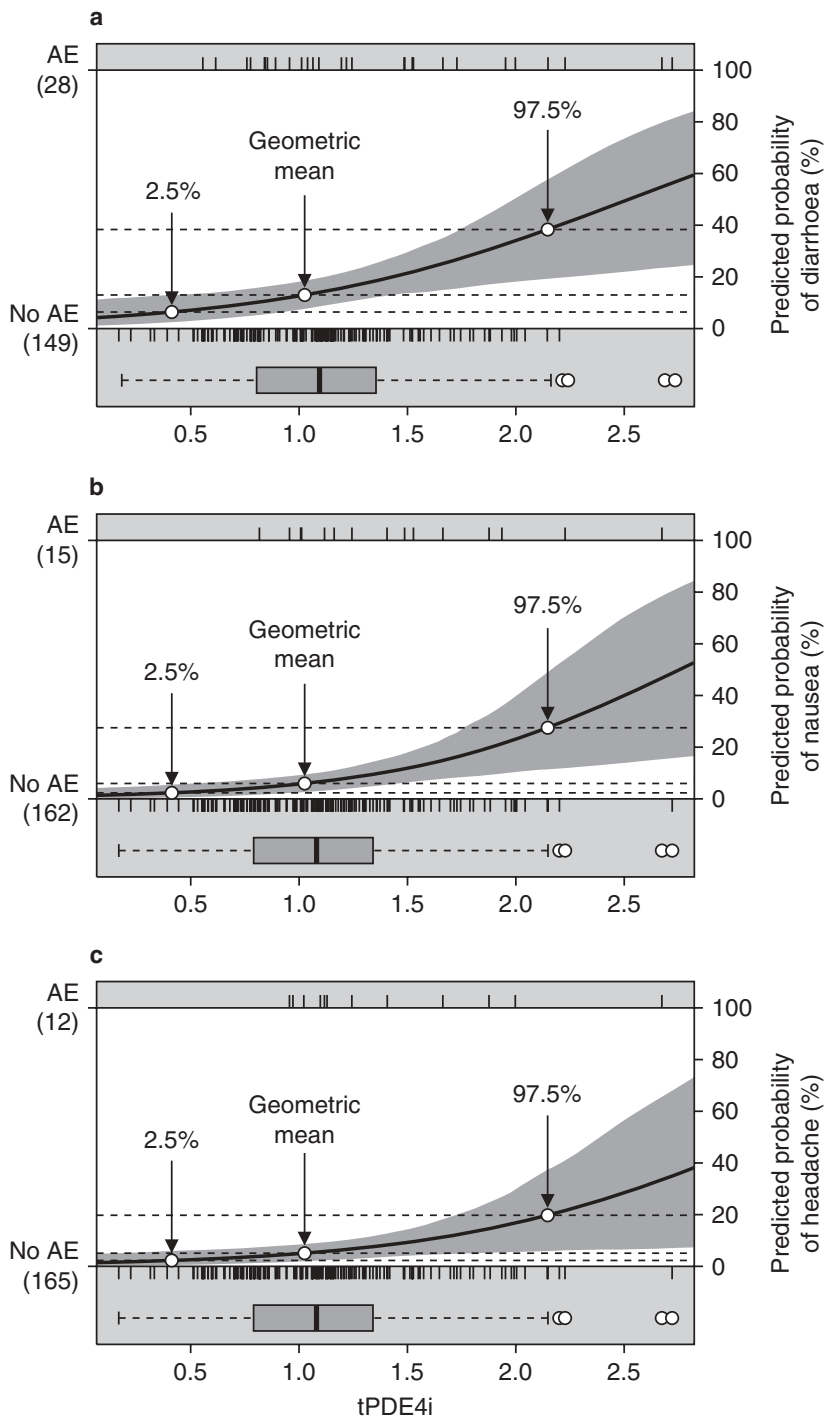

Fig. 3. Logistic regression models to predict the probability and/or frequency of developing (a) diarrhoea, (b) nausea or (c) headache, with total phosphodiesterase-4 inhibitory (tPDE4i) activity as the exposure variable. Each vertical bar represents a subject with either at least one adverse event (AE) [upper thin solid line] or with no AE [lower thin solid line]; the bars are distributed along the horizontal axes according to the tPDE4i for each subject. The sigmoid-shaped curves represent the logistic models. A nonparametric bootstrap procedure was used to estimate the $95 \%$ Cls (dark grey shaded areas) of the mean model predictions (thick solid black lines). Model predictions for TPDE4i are highlighted at the $2.5 \%$ and $97.5 \%$ quantiles, with the geometric means also represented (dashed lines) [see also table IV]. The box and whisker plots ${ }^{[35]}$ present the tPDE4i values used for modelling; the boxes represent the medians and the 25th (Q1) and 75th (Q3) percentiles, the whiskers represent values 1.5 times the Q1-Q3 values, and the white circles represent the outliers.

to sex-differential effects on CYP1A2. CYP1A2 activity is lower in women, which is mainly attributed to differential regulation of CYP1A2 during the course of the menstrual cycle, ${ }^{[15]}$ lower activity has also been shown in liver microsomes from women. ${ }^{[40]}$ The sex differences described in this report were consistent with the greater bodyweight in men than in women. Stability of the full model for roflumilast was achieved by removing bodyweight and alcohol consumption from the expression for clearance. Bodyweight may have competed with the effect of sex on CYP1A2 to explain the same interindividual variability in clearance, thus explaining some of the observed instability prior to its removal as a covariate. Considering the reports of the differential effects of sex on CYP1A2 activity, the removal of bodyweight from the expression for clearance was justified.

Age-associated changes in body composition may also affect the $\mathrm{V}_{\mathrm{d}}$ or $\mathrm{Q}$ of a drug. ${ }^{[11]}$ Although CYP activity in human liver microsomes, cultured hepatocytes and liver slices is not affected by the age of the donor, ${ }^{[39,42]}$ CYP2C19 activity shows a decrease with age and has higher interindividual variability in older subjects. ${ }^{[15]}$ In the present analysis, differences in the clearance of roflumilast were not associated with age, whereas clearance of roflumilast $\mathrm{N}$-oxide was estimated to decrease by $25.6 \%$ between the ages of 40 and 75 years. CYP2C19 may therefore be more important in eliminating roflumilast $N$-oxide than roflumilast. Age was also associated with changes in blood perfusion of the liver and kidneys, but the lack of an age effect on roflumilast suggests that the age effect on roflumilast $\mathrm{N}$-oxide was not the result of changes in hepatic perfusion and may instead have been due to a decrease in the $V_{1}$. Age, however, was not evaluated as a covariate on the $\mathrm{V}_{1}$ values of either analyte.

Both models for roflumilast and roflumilast $N$-oxide predicted higher exposure in Black and Hispanic subjects than in White subjects. The two models differed in the way that race covariates were incorporated. Covariates representing race effects were included in the expression of clearance for roflumilast and in the expression of the $F_{\text {rel }}$ for roflumilast $N$-oxide. In both cases, the apparent clearance $\left(\mathrm{CL} / \mathrm{F}_{\text {rel }}\right)$ was predicted to be lower in Black and Hispanic subjects. These model predictions were in concordance with the results of NCAs and were corroborated by similar findings of decreased intrinsic clearance of midazolam (a CYP3A4 substrate) in Black subjects ${ }^{[43]}$ and decreased 7-ethoxyresorufin $O$-dealkylase activity (a measure of CYP1A2) in hepatic microsomes from Hispanic donors. ${ }^{[40]}$ However, one limitation of the current study was that the impact of Hispanic race on the disposition of roflumilast and roflumilast $N$-oxide could not be determined with high precision. Uncertainty around this covariate was greater than for the other covariates because of the limited data available.

The effects of smoking in increasing the clearance of roflumilast and roflumilast $N$-oxide may be explained by greater 
CYP1A2 activity. Caffeine metabolite ratios indicate that CYP1A2 activity is $79 \%$ greater in cigarette smokers than in non-smokers. ${ }^{[17]}$ An increase in CYP1A2 activity would therefore be expected to directly increase the clearance of roflumilast. The apparent increase in the clearance of roflumilast $N$-oxide suggests either that roflumilast $N$-oxide is a substrate for CYP1A2 or that CYP1A2 may preferentially catalyse the formation of a different metabolite, thus reducing the proportion of roflumilast that is converted to the $N$-oxide metabolite via the CYP3A4 pathway. This would decrease the $\mathrm{F}_{\text {rel }}$ of roflumilast $N$-oxide and manifest in an increase in $\mathrm{CL} / \mathrm{F}_{\text {rel }}$.

Estimates of the pharmacokinetic parameters of roflumilast and roflumilast $N$-oxide differed between COPD patients and healthy subjects. The estimated covariate effect of COPD on roflumilast clearance indicated a reduction of $39 \%$. The typical clearance in COPD patients would be $6.36 \mathrm{~L} / \mathrm{h}$, compared with $10.5 \mathrm{~L} / \mathrm{h}$ in healthy subjects. The estimated $\mathrm{V}_{1}$ of roflumilast was $184 \%$ larger in COPD patients than in healthy subjects. The typical $\mathrm{V}_{1}$ for COPD patients would be $40.6 \mathrm{~L}$, compared with $14.3 \mathrm{~L}$ in healthy subjects. The differences for roflumilast $N$ oxide were less pronounced. The estimated $\mathrm{V}_{\mathrm{d}}$ was $21 \%$ smaller, and clearance was $7.9 \%$ lower in COPD patients than in healthy subjects. The differences in the $\mathrm{V}_{\mathrm{d}}$ estimates for roflumilast and roflumilast $N$-oxide are likely to be influenced by the frequency of pharmacokinetic sampling, as sparse sampling carries uncertainty. This uncertainty would be more pronounced with roflumilast, which had a two-compartment model, than with roflumilast $N$-oxide, which had a one-compartment model.

COPD is associated with inflammatory markers that are released in response to ongoing chronic lung inflammation, including interleukin (IL)-1, IL-2 and IL-6 and tumour necrosis factor (TNF)- $\alpha$. These markers are present either permanently or transiently in the systemic circulation and, as such, may exert their action beyond the lungs. ${ }^{[44]}$ IL- 6 and TNF $\alpha$ are known to be increased in the systemic circulation of COPD patients, particularly during exacerbations, ${ }^{[4,46]}$ and they increase muscle weakness and atrophy. In addition, these inflammatory markers downregulate CYP3A4 and CYP1A2, which are responsible for roflumilast metabolism. ${ }^{[47]}$ These effects could explain, to a large extent, the differences we observed between healthy subjects and patients with COPD. Overall, it appears that the inflammatory markers present in COPD are responsible for the reduction in clearance of roflumilast and roflumilast $N$-oxide via the depressive effect and downregulation of CYP enzymes.

Estimates of tPDE4i exposure in the different population subgroups included in this analysis showed that, among the possible combinations of covariates for healthy subjects, elderly (80-year-old), Black, non-smoking females would be expected to have the highest tPDE4i values compared with the values in the reference population (40-year-old, White, male, non-smoking, healthy subjects). Their geometric mean tPDE4i [95\% CI] value would be increased to $194 \%[94,401]$ (i.e. a $94 \%$ $[-6,301]$ higher geometric mean tPDE4i). Conversely, 40-yearold, White, male, healthy subjects who smoked would be expected to have the lowest relative mean tPDE4i value, with a decrease to $81 \%$ [40, 165] (i.e. $19 \%$ [-60, 65] lower tPDE4i) compared with the reference population value. Thus, all subgroups of healthy subjects in this analysis would be expected to have mean tPDE4i values (relative to those of the reference population) of between approximately $-20 \%$ and $+100 \%$. Inclusion of patients with COPD increases the upper limit of expected tPDE4i values; the expected values $[95 \% \mathrm{CI}]$ in elderly, Black, female, non-smoking, COPD patients would be $217 \%$ $[107,437]$ (i.e. $117 \%[7,337]$ higher tPDE4i) compared with the reference population value. The high degree of variability of this estimate reflects the relatively small population of patients used to derive these data. Elderly, non-smoking, Black, female patients were poorly represented in the modelling and validation datasets; indeed, they were poorly represented across the clinical trial programme. This limitation must be remembered when interpreting the COPD results. In the total COPD safety pool, comprising 12054 patients, there were 29 Black, nonsmoking, female patients of any age (13 who received placebo and 16 who received roflumilast; unpublished data). Of these, 21 reported AEs (12 in the roflumilast group and 9 in the placebo group), and the rate of AEs was no different from that in the rest of the population.

The phase I programme for roflumilast did not raise any safety signals for coadministration with a variety of drugs, ${ }^{[11-13,18,21,48-55]}$ in smokers, ${ }^{[56]}$ in hepatically ${ }^{[19]}$ or renally ${ }^{[55]}$ impaired patients, or after intake with food. ${ }^{[10]}$ Haematological and clinical chemistry parameters were not affected to any significant degree. Because some of these agents act as metabolic inhibitors or inducers, their action on the CYP isoenzymes that metabolize roflumilast would be expected to alter tPDE4i during coadministration. Using our models, the effect of any change in tPDE4i on the predicted probabilities of nausea, diarrhoea or headache can be assessed.

We used the model to investigate whether tPDE4i could predict the occurrence of AEs. AEs occurring significantly more frequently in patients treated with roflumilast than in patients receiving placebo were analysed by logistic regression, and models were successfully established for diarrhoea, nausea and headache. The exposure variables $\mathrm{AUC}_{\mathrm{m}}$ and $\mathrm{tPDE} 4 \mathrm{i}$ demonstrated similar precision in predicting the probability of 
a patient developing these events, whereas the exposure variable $\mathrm{AUC}_{\mathrm{p}}$ showed a much weaker association with AEs in all PT categories. There was, however, good agreement among all three exposure variables in the predicted probability of developing AEs in the reference population. Based on the geometric mean tPDE4i of the reference population, a patient would have a $13 \%$ (95\% CI $7.5,18.5)$ probability of developing diarrhoea, a $6.0 \%(95 \%$ CI $2.6,9.4)$ probability of developing nausea, and a $5.1 \%$ (95\% CI 1.9, 8.6) probability of developing headache. At the upper limit of the 95\% CI, the tPDE4i was approximately double the geometric mean value (2.15 vs 1.03 ). At this extreme value, the models predicted probabilities of $38.3 \%$ for developing diarrhoea, $27.5 \%$ for nausea and $19.8 \%$ for headache. However, at such extreme values, model predictions have a high level of uncertainty and thus need to be interpreted with care.

It is useful to compare the $\mathrm{AE}$ modelling results with those obtained from the more recent, and far larger, placebocontrolled trials of roflumilast, in which a total of 4767 patients were randomized and treated. Thus, in the 12-month trials in patients with severe COPD with bronchitis symptoms (M2-124 and $\mathrm{M} 2-125)^{[7]}$ and the 6-month trials in patients with moderate-to-severe COPD who were using concomitant long-acting bronchodilators (M2-127 and M2-128), ${ }^{[8]}$ the incidence of AEs was lower than the current model predictions at the geometric mean tPDE4i. Thus, across the trials, the incidence of diarrhoea as an $\mathrm{AE}$ in patients receiving roflumilast was $8-9 \%$, the incidence of nausea was $3-5 \%$ and the incidence of headache was $2-3 \%$, compared with $<1-3 \%,<1-2 \%$ and $0-2 \%$, respectively, in those receiving placebo. ${ }^{[7,8]}$ The higher incidence of AEs with roflumilast led to higher withdrawal rates over the entire duration of the trials, with $14 \%$ of roflumilast-treated patients withdrawing because of an $\mathrm{AE}$, versus $11 \%$ of those on placebo (pooled M2-124 and M2-125 data), ${ }^{[7]} 17 \%$ versus $10 \%$ (M2-127) and $9 \%$ versus 5\% (M2-128). ${ }^{[8]}$ However, after week 12 of therapy, withdrawals were similar between the roflumilast and placebo groups (9\% vs 9\%, respectively [pooled M2-124 and M2-125 data]). ${ }^{[7]}$

In the phase III study that provided the AE data used for modelling (M2-110), ${ }^{[29]}$ there was no difference in the reporting of weight loss as an AE between the groups; for this reason, weight loss was not included in our AE model. Unfortunately, pharmacokinetic samples were not available from the M2-124, M2-125, M2-127 and M2-128 studies, in which weight loss was seen, and so these data cannot be analysed with our models. For completeness, we have summarized the weight loss information below. In the pooled M2-124 and M2-125 safety analysis, the mean $[\mathrm{SD}$ ] weight change was greater in patients receiving active treatment $(-2.1[4.0] \mathrm{kg})$ than in patients receiving placebo $(0.1[3.5] \mathrm{kg})$. In the roflumilast group, the weight loss was greater if the patient had experienced at least one gastrointestinal AE or headache $(-2.6$ [3.7] kg, $\mathrm{n}=196)$ than if they had not $(-2.0$ [4.0] $\mathrm{kg}, \mathrm{n}=1302)$ and it was greater in heavier patients (e.g. -0.7 [2.5] kg in patients with a body mass index $[\mathrm{BMI}] \leq 18 \mathrm{~kg} / \mathrm{m}^{2}$ and $-3.6[5.5] \mathrm{kg}$ in those with a BMI $\left.>30 \mathrm{~kg} / \mathrm{m}^{2}\right) .{ }^{[7]}$ Similar results were seen in the M2-127 and M2128 studies. ${ }^{[8]}$

\section{Conclusions}

The pharmacokinetics of roflumilast can be modelled adequately with a two-compartment model with first-order absorption including a $t_{\text {lag. }}$. The covariates sex, smoking and race influenced clearance; food influenced the $k_{a}$ and $t_{\text {lag. }}$. The pharmacokinetics of the roflumilast metabolite, roflumilast $\mathrm{N}$-oxide, can be modelled adequately with a one-compartment model with zero-order absorption. The covariates age, sex and smoking influenced clearance; age, sex and race influenced the $\mathrm{F}_{\text {rel }}$; bodyweight influenced the $\mathrm{V}_{\mathrm{d}}$; and food influenced the apparent duration of formation. Posterior predictive checks and robustness analysis showed that the population pharmacokinetic models for roflumilast and roflumilast $\mathrm{N}$-oxide adequately described the pharmacokinetic parameters and the covariate effects on disposition.

Modelling-based inferences of the effects of the covariates on exposure were supported by data-derived descriptive statistics on the noncompartmental $\mathrm{AUC}_{24}$ at steady state. These results corroborated very well with the current understanding of the role of inflammatory cytokines in the regulation of CYPs.

The final models in healthy subjects were used to estimate the exposure of roflumilast and roflumilast $N$-oxide in the COPD patient population. The model predicted an effect of COPD on roflumilast clearance $\left(\theta_{22}\right)$ of -0.394 and on roflumilast $N$-oxide clearance $\left(\theta_{25}\right)$ of -0.0785 . Calculating exposure as (equation 9):

$$
\text { Exposure }=\frac{\text { Dose }}{\text { Clearance } \times(1+\theta)}
$$

these estimates gave a COPD effect on roflumilast exposure of 1.65 (an increase of 65\%) and a COPD effect on roflumilast $N$ oxide exposure of 1.085 (an increase of $8.5 \%$ ). tPDE4i, which included combined exposure to both roflumilast and roflumilast $N$-oxide, was seen to increase by approximately $13 \%$, reflecting the larger contribution of roflumilast $N$-oxide than that of roflumilast. When additional covariates were included in the tPDE4i calculation, the expected tPDE4i values increased by a maximum of $117 \%(95 \%$ CI 7,337$)$ compared with 
those of the reference population (e.g. in an elderly, nonsmoking, Black woman with COPD). The high degree of variability of this estimate reflected the relatively small population of patients used to derive these data.

Population models showed a general association between tPDE4i and the occurrence of common AEs. We suggest that the concept of tPDE4i can be used to evaluate potential precautions or clinical situations (e.g. coadministration with metabolic inhibitors or inducers). Our models may therefore be used to assess, in a quantifiable way, the probability of tolerability problems occurring, and may provide a valuable aid to riskbenefit assessments, especially in the early stages of treatment.

\section{Acknowledgements}

The study was supported by Nycomed GmbH (Konstanz, Germany). The authors thank Brian Sadler (Strategic PK Consulting, Cary, NC, USA) for his contribution to the extension of the phase I population pharmacokinetic models to COPD patients, Peter Van Ess for his support in several phase I studies, and Polly Field and Jonathan Weeks (Caudex Medical Ltd, Oxford, UK) for editorial assistance with the preparation of the manuscript.

Funding for the design and conduct of this study; collection, management, analysis and interpretation of the data; and preparation, review and approval of the manuscript was provided by Nycomed GmbH. Gezim Lahu, Andreas Hünnemeyer, Edgar Diletti, Martin Elmlinger, Nigel McCracken and Axel Facius are employees of Nycomed GmbH. Karl Zech serves as a consultant to Nycomed $\mathrm{GmbH}$ and was an employee of the company at the time when the study was conducted. Peter Ruth has no conflicts of interest to declare.

\section{References}

1. Hatzelmann A, Schudt C. Anti-inflammatory and immunomodulatory potential of the novel PDE4 inhibitor roflumilast in vitro. J Pharmacol Exp Ther 2001; 297 (1): 267-79

2. Jones NA, Boswell-Smith V, Lever R, et al. The effect of selective phosphodiesterase isoenzyme inhibition on neutrophil function in vitro. Pulm Pharmacol Ther 2005; 18 (2): 93-101

3. Bundschuh DS, Eltze M, Barsig J, et al. In vivo efficacy in airway disease models of roflumilast, a novel orally active PDE4 inhibitor. J Pharmacol Exp Ther 2001; 297 (1): 280-90

4. Grootendorst DC, Gauw SA, Verhoosel RM, et al. Reduction in sputum neutrophil and eosinophil numbers by the PDE4 inhibitor roflumilast in patients with COPD. Thorax 2007; 62 (12): 1081-7

5. Calverley PM, Sanchez-Toril F, McIvor A, et al. Effect of 1-year treatment with roflumilast in severe chronic obstructive pulmonary disease. Am J Respir Crit Care Med 2007; 176 (2): 154-61

6. Rabe KF, Bateman ED, O'Donnell D, et al. Roflumilast - an oral antiinflammatory treatment for chronic obstructive pulmonary disease: a randomised controlled trial. Lancet 2005; 366 (948): 563-71

7. Calverley PM, Rabe KF, Goehring UM, et al. Roflumilast in symptomatic chronic obstructive pulmonary disease: two randomised clinical trials. Lancet 2009; 374 (9691): 685-94

8. Fabbri LM, Calverley PM, Izquierdo-Alonso JL, et al. Roflumilast in moderateto-severe chronic obstructive pulmonary disease treated with longacting bronchodilators: two randomised clinical trials. Lancet 2009; 374 (9691): 695-703

9. Bethke TD, Böhmer GM, Hermann R, et al. Dose-proportional intraindividual single- and repeated-dose pharmacokinetics of roflumilast, an oral, once-daily phosphodiesterase 4 inhibitor. J Clin Pharmacol 2007; 47 (1): 26-36

10. Hauns B, Hermann R, Hünnemeyer A, et al. Investigation of a potential food effect on the pharmacokinetics of roflumilast, an oral, once-daily phosphodiesterase 4 inhibitor, in healthy subjects. J Clin Pharmacol 2006; 46 (10): 1146-53

11. Lahu G, Hünnemeyer A, Herzog R, et al. Effect of repeated dose of erythromycin on the pharmacokinetics of roflumilast and roflumilast $\mathrm{N}$-oxide. Int J Clin Pharmacol Ther 2009; 47 (4): 236-45

12. Lahu G, Hünnemeyer A, von Richter $\mathrm{O}$, et al. Effect of single and repeated doses of ketoconazole on the pharmacokinetics of roflumilast and roflumilast N-oxide. J Clin Pharmacol 2008; 48 (11): 1339-49

13. von Richter O, Lahu G, Hünnemeyer A, et al. Effect of fluvoxamine on the pharmacokinetics of roflumilast and roflumilast N-oxide. Clin Pharmacokinet 2007; 46 (7): 613-22

14. Cotreau MM, von Moltke LL, Greenblatt DJ. The influence of age and sex on the clearance of cytochrome P450 3A substrates. Clin Pharmacokinet 2005; 44 (1): 33-60

15. Bebia Z, Buch SC, Wilson JW, et al. Bioequivalence revisited: influence of age and sex on CYP enzymes. Clin Pharmacol Ther 2004; 76 (6): 618-27

16. Mangoni AA, Jackson SH. Age-related changes in pharmacokinetics and pharmacodynamics: basic principles and practical applications. Br J Clin Pharmacol 2004; 57 (1): 6-14

17. Funck-Brentano C, Raphael M, Lafontaine M, et al. Effects of type of smoking (pipe, cigars or cigarettes) on biological indices of tobacco exposure and toxicity. Lung Cancer 2006; 54 (1): 11-8

18. Hermann R, Siegmund W, Giessmann T, et al. The oral, once-daily phosphodiesterase 4 inhibitor roflumilast lacks relevant pharmacokinetic interactions with inhaled budesonide. J Clin Pharmacol 2007; 47 (8): 1005-13

19. Hermann R, Nassr N, Lahu G, et al. Steady-state pharmacokinetics of roflumilast and roflumilast $\mathrm{N}$-oxide in patients with mild and moderate liver cirrhosis. Clin Pharmacokinet 2007; 46 (5): 403-16

20. David M, Zech K, Seiberling M, et al. Roflumilast, a novel, oral, selective PDE4 inhibitor, shows high oral bioavailability. J Allergy Clin Immunol 2004; 113: S220-1

21. Nassr N, Lahu G, Hünnemeyer A, et al. Magnesium hydroxide/aluminium hydroxide-containing antacid does not affect the pharmacokinetics of the targeted phosphodiesterase 4 inhibitor roflumilast. J Clin Pharmacol 2007; 47 (5): 660-6

22. Hermann R, Lahu G, Hauns B, et al. 'Total PDE4 inhibitory activity': a concept for evaluating pharmacokinetic alterations of roflumilast and roflumilast N-oxide in special populations and drug-drug interactions [abstract no. 2542]. Eur Respir J 2006; 28 Suppl. 50: 436s [online]. Available from URL: http:// www.ers-education.org/pages/default.aspx?id=335\&idBrowse $=7697 \&$ det $=1$ [Accessed 2010 Jun 14]

23. Manning CD, Burman M, Christensen SB, et al. Suppression of human inflammatory cell function by subtype-selective PDE4 inhibitors correlates with inhibition of PDE4A and PDE4B. Br J Pharmacol 1999; 128 (7): 1393-8

24. Halpin DM. ABCD of the phosphodiesterase family: interaction and differential activity in COPD. Int J Chron Obstruct Pulmon Dis 2008; 3 (4): 543-61

25. Lipworth BJ. Phosphodiesterase-4 inhibitors for asthma and chronic obstructive pulmonary disease. Lancet 2005; 365 (9454): 167-75

26. Spina D. Phosphodiesterase-4 inhibitors in the treatment of inflammatory lung disease. Drugs 2003; 63 (23): 2575-94

27. Spina D. PDE4 inhibitors: current status. Br J Pharmacol 2008; 155 (3): 308-15

28. Kowalski KG, Hutmacher MM. Efficient screening of covariates in population models using Wald's approximation to the likelihood ratio test. J Pharmacokinet Pharmacodyn 2001; 28 (3): 253-75 
29. Altana Pharma. Effect of roflumilast on pulmonary function and respiratory symptoms in patients with chronic obstructive pulmonary disease (COPD) (BY217/M2-110) [ClinicalTrials.gov identifier NCT00062582]. US National Institutes of Health, ClinicalTrials.gov [online]. Available from URL: http:// www.clinicaltrials.gov/ct2/show/NCT00062582?id=NCT00062582\&rank=1 [Accessed 2010 Jun 14]

30. Data on file, Nycomed GmbH, 2009

31. Efron B, Tibshirani RJ. An introduction to the bootstrap. Boca Raton (FL): Chapman and Hall/CRC, 1993

32. Wang DD, Zhang S. Standardized visual predictive check: how and when to use it model validation [abstract no. 1501]. 18th Annual Meeting, Population Approach Group in Europe; 2009 Jun 23-26; St Petersburg [online]. Available from URL: http://www.page-meeting.org/default.asp?abstract=1501 [Accessed 2010 Jun 14]

33. Maintenance and Support Services Organization. The medical dictionary for regulatory activities. Chantilly (VA): Northrop Grumman Corporation, 2010

34. Egan J. Signal detection theory and ROC analysis. New York: Academic Press, 2010

35. Chambers JM, Cleveland WS, Kleiner B, et al. Graphical methods for data analysis. Boston (MA): Wadsworth \& Brooks/Cole, 1983: 62

36. Bonate P. Pharmacokinetic-pharmacodynamic modeling and simulation. New York: Springer, 2006

37. Harris RZ, Benet LZ, Schwartz JB. Gender effects in pharmacokinetics and pharmacodynamics. Drugs 1995; 50 (2): 222-39

38. McCune JS, Lindley C, Decker JL, et al. Lack of gender differences and large intrasubject variability in cytochrome $\mathrm{P} 450$ activity measured by phenotyping with dextromethorphan. J Clin Pharmacol 2001; 41 (7): 723-31

39. Hunt CM, Westerkam WR, Stave GM. Effect of age and gender on the activity of human hepatic CYP3A. Biochem Pharmacol 1992; 44 (2): 275-83

40. Parkinson A, Mudra DR, Johnson C, et al. The effects of gender, age, ethnicity, and liver cirrhosis on cytochrome $\mathrm{P} 450$ enzyme activity in human liver microsomes and inducibility in cultured human hepatocytes. Toxicol Appl Pharmacol 2004; 199 (3): 193-209

41. Strum DP, Eger EI, Unadkat JD, et al. Age affects the pharmacokinetics of inhaled anesthetics in humans. Anesth Analg 1991; 73 (3): 310-8

42. Wynne HA, Mutch E, James OF, et al. The effect of age upon the affinity of microsomal mono-oxygenase enzymes for substrate in human liver. Age Ageing 1988; 17 (6): 401-5
43. Wandel C, Witte JS, Hall JM, et al. CYP3A activity in African American and European American men: population differences and functional effect of the CYP3A4*1B5'-promoter region polymorphism. Clin Pharmacol Ther 2000; 68 (1): $82-91$

44. Fabbri LM, Rabe KF. From COPD to chronic systemic inflammatory syndrome? Lancet 2007; 370 (9589): 797-9

45. Bhowmik A, Seemungal TA, Sapsford RJ, et al. Relation of sputum inflammatory markers to symptoms and lung function changes in COPD exacerbations. Thorax 2000; 55 (2): 114-20

46. Tracey KJ, Wei H, Manogue KR, et al. Cachectin/tumor necrosis factor induces cachexia, anemia, and inflammation. J Exp Med 1988; 167 (3): 1211-27

47. Masubuchi Y, Enoki K, Horie T. Down-regulation of hepatic cytochrome P450 enzymes in rats with trinitrobenzene sulfonic acid-induced colitis. Drug Metab Dispos 2008; 36 (3): 597-603

48. Nassr N, Lahu G, von Richter O, et al. Lack of a pharmacokinetic interaction between steady-state roflumilast and single-dose midazolam in healthy subjects. Br J Clin Pharmacol 2007; 63 (3): 365-70

49. Data on file, Nycomed GmbH, 2007

50. Böhmer GM, Nassr N, Wenger M, et al. The targeted oral, once-daily phosphodiesterase 4 inhibitor roflumilast and the leukotriene receptor antagonist montelukast do not exhibit significant pharmacokinetic interactions. J Clin Pharmacol 2009; 49 (4): 389-97

51. Bethke TD, Giessmann T, Westphal K, et al. Roflumilast, a once-daily oral phosphodiesterase 4 inhibitor, lacks relevant pharmacokinetic interactions with inhaled salbutamol when co-administered in healthy subjects. Int $\mathbf{J}$ Clin Pharmacol Ther 2006; 44 (11): 572-9

52. Nassr N, Hünnemeyer A, Herzog R, et al. Effects of rifampicin on the pharmacokinetics of roflumilast and roflumilast $\mathrm{N}$-oxide in healthy subjects. Br J Clin Pharmacol 2009; 68 (4): 580-7

53. Data on file, Nycomed GmbH, 2005

54. Data on file, Nycomed GmbH, 2002

55. Data on file, Nycomed GmbH, 2001

56. Data on file, Nycomed GmbH, 2000

Correspondence: Gezim Lahu, Nycomed GmbH, Department of Pharmacometrics and Pharmacokinetics, Byk-Gulden-Str. 2, 78647 Konstanz, Germany.

E-mail: gezim.lahu@nycomed.com 